\title{
Ethnobotanical survey of the medicinal flora of Harighal, Azad Jammu \& Kashmir, Pakistan
}

\author{
Muhammad Shoaib Amjad ${ }^{1 *}$, Ujala Zahoor', Rainer W. Bussmann², Muhammad Altaf ${ }^{3}$, \\ Syed Mubashar Hussain Gardazi ${ }^{1}$ and Arshad Mehmood Abbasi ${ }^{4^{*}}$
}

\begin{abstract}
Background: The present study is the first quantitative ethnobotanical evaluation of Harighal, an inaccessible and unexplored area of District Bagh Azad Jammu \& Kashmir (AJK). The exploration, quantification, and comparison of ethnobotanical knowledge among different rural communities of the study area were mainly focused during field survey.

Methodology: In total, 79 informants (49 men and 34 women) were selected randomly to collect data using a semistructured questionnaire. Various quantitative indices, including use value, relative frequency of citation, relative importance, fidelity level, and informant consent factor, were employed to evaluate the gathered information. Furthermore, primary data were also compared with twenty-two papers published from adjoining areas.

Result: A total of 150 medicinal plants belonging to 98 genera and 60 families were documented. Asteraceae, Fabaceae, and Rosaceae were the dominant families having 15 species each. Of these, 76 species were indigenous, 74 exotic, 136 were collected in the wild, 10 cultivated, and 4 both wild-collected and cultivated. Herbaceous taxa were the most used life form, and leaves were the most exploited plant part. Decoctions were the most preferred method used in preparation of herbal recipes. Three species viz. Mentha longifolia, Berberis lycium, and Galium aparine had the highest use value (1.05), relative frequency of citation (0.81), and relative importance value (96), respectively. The highest informant consensus factor (ICF) was reported for digestive disorders. Mentha longifolia, Punica granatum, Zanthoxylum alatum, and Olea ferruginea had $100 \%$ fidelity values. The Jaccard index revealed that uses of plants were more similar in two neighboring areas, i.e., Pearl Valley and Toli Peer.

Conclusion: Local inhabitants still prioritize herbal medicines as an effective way to treat a wide variety of ailments. Elders and health practitioners of the study area are well aware of indigenous knowledge about medicinal plants, but young people are not much interested in herbal practices. Thus, valuable knowledge about the use of plants is on the verge of decline.
\end{abstract}

Keywords: Ethnobotany, Medicinal flora, Used value, Fidelity level, Azad Jammu \& Kashmir, Pakistan

* Correspondence: malikshoaib1165@yahoo.com; arshad799@yahoo.com ${ }^{1}$ Department of Botany, Women University of Azad Jammu \& Kashmir, Bagh 12500, Pakistan

${ }^{4}$ Department of Environmental Sciences, COMSATS University Islamabad, Abbottabad Campus, Abbottabad 22060, Pakistan

Full list of author information is available at the end of the article

\section{Background}

Ethnobotanical surveys focus on the complex connection between local inhabitants and local plants, including practices and cultural beliefs associated with different forms of uses [1-4]. These studies are important in highlighting the value of native plant species, e.g., for discovering novel drugs [5]. Medicinal plants are

(c) The Author(s). 2020 Open Access This article is licensed under a Creative Commons Attribution 4.0 International License, which permits use, sharing, adaptation, distribution and reproduction in any medium or format, as long as you give appropriate credit to the original author(s) and the source, provide a link to the Creative Commons licence, and indicate if changes were made. The images or other third party material in this article are included in the article's Creative Commons licence, unless indicated otherwise in a credit line to the material. If material is not included in the article's Creative Commons licence and your intended use is not permitted by statutory regulation or exceeds the permitted use, you will need to obtain permission directly from the copyright holder. To view a copy of this licence, visit http://creativecommons.org/licenses/by/4.0/ The Creative Commons Public Domain Dedication waiver (http://creativecommons.org/publicdomain/zero/1.0/) applies to the data made available in this article, unless otherwise stated in a credit line to the data. 
imperative for the livelihoods of underprivileged communities across the world [6-11]. Globally, 35,000-70, 000 plant species are used in folk medicine [12]. In developing countries, $60-80 \%$ of the population is still relying on plant-based medicines because they are economical and safe alternative to often inaccessible allopathic medicine $[13,14]$. Even in the developed world, herbal remedies are extensively used, e.g., $30-50 \%$ of the population in China, $40-50 \%$ in Germany, $48 \%$ in Australia, $42 \%$ in the USA, and $49 \%$ in France reported using herbal medicine as supplementary health care [15-17]. About $25 \%$ of modern allopathic drugs are derived directly from plants or synthetic analogues of different compounds isolated from medicinal plants [18]. Plant-based drugs are effective and often have less side effects. This can be best explained by comparison between the extract bark of important medicinal plant Salix alba (white willow) with the synthetic drug aspirin which has more reported side effects. Different studies confirmed that extract of Salix alba bark can avoid the side effects caused by aspirin [19].

The traditional knowledge of medicinal plants is held by many rural communities even in our times [20-24]. Such knowledge is transmitted from generation to generation [5, 25-27]. Differences and similarities in traditional knowledge and practice among two different cultural groups living within the same ecological region are fascinating, as they can provide understanding of how cultural reflection can change individual viewpoints about the environment and also guide interactions between human beings and resources in the ecosystem [28]. However, traditional knowledge on plant species is decreasing gradually across the globe [29]. This knowledge is usually held by hakims (traditional healers) and elderly people and be passed to the next generation via verbal communication only [30]; thus, there is a serious danger of knowledge loss due to the progression in the modern health care system, rapid urbanization, and poor relations between younger and old generation [31-34]. The documentation of traditional ethnomedicinal knowledge is of high importance and may contribute to the development of new drugs. Furthermore, this may also contribute to the maintenance of indigenous culture and natural resource management.

Pakistan has a large wealth of medicinal and aromatic plants due to its diverse habitat, climate, and soil types and harbors about 6000 wild plant species [35]. Among them, 400-600 species are used for therapeutic purposes. Eighty percent of this medicinal flora is restricted in Northwestern areas of Pakistan and Azad Kashmir [36-38]. In the early 1950s, 84\% of population of Pakistan depended upon plants for treating various ailments; but nowadays, this practice is restricted to remote areas due to modernization and rapid change in lifestyle [39]. Previously, different ethnobotanical studies were conducted to document the traditional knowledge about medicinal plants and herbal recipes in remote areas of Pakistan and Azad Jammu \& Kashmir [40-44]. However, Tehsil Harighal of District Bagh is still unexplored ethnobotanically, especially due to topographical challenges like hilly terrain and steep slopes, and cultural and religious restrictions that limits researcher access to document ethnobotanical knowledge. We hypothesized that due to the remoteness of the area, the ethnobotanical knowledge of Harighal would considerably differ from other areas of Pakistan. This study was planned with the objective to document the indigenous knowledge about medicinal plants used for primary health care particularly focusing on methods of preparation and administration of herbal recipes. The data was further analyzed by using various numerical indices and compared with previous studies to determine the novelty of work.

\section{Materials and methods}

\section{Study area}

Harighal $\left(33^{\circ} 54^{\prime} 34^{\circ} 08^{\prime} \mathrm{N}\right.$ to $\left.73^{\circ} 01^{\prime} 73^{\circ} 38^{\prime} \mathrm{E}\right)$, a Tehsil of District Bagh, is located in western Himalayan foothills of Pirpanjal, with altitude ranges between 900 and $2300 \mathrm{~m}$ (Fig. 1) [45]. It is $155 \mathrm{~km}$ away from Islamabad, the capital of Pakistan and $98 \mathrm{~km}$ away from the Muzaffarazad, the capital of Azad Jammu \& Kashmir. The total area of Harighal is $712 \mathrm{~km}^{2}$ and its population is about 120,000 according to the 2017 census. The climate is subtropical-temperate with about $1500 \mathrm{~mm}$ average annual precipitation. The summers are hot with temperature ranges between 21 and $40{ }^{\circ} \mathrm{C}$ while winters is cold with temperatures around $2{ }^{\circ} \mathrm{C}$ during January (Fig. 2) [46]. The vegetation is mainly dominated by Olea ferruginea at lower altitudes, Pinus roxbughaii and Quercus incana at mid altitudes, and Pinus wallichiana at higher altitude of the forest belt. Most of the area is occupied by open grassland.

The region has a diverse and complex ethnic composition with Rajputs, Maldial, Sudhan, Khawaja, Gujar, and Jat. Rajput tribes spread across the region and the Maldial tribe is regarded as the most influential ethnic group in Harighal, Azad Kashmir. Almost all of the inhabitants are Muslims. Popular languages are Pahari, Hindko, Gojri, and Kashmiri, but most inhabitants are also familiar with Urdu.

The area is remote, with difficult mountainous terrain and quite far from urban centers. Local inhabitants have poor socioeconomic conditions, lacking government services, and modern healthcare facilities. The roads and other infrastructure are poorly developed, and many inhabitants are dedicated to agriculture, livestock, and their own small-scale business. Some are educated and are government servants, while very few are serving abroad. Agriculture is mainly dependent upon rain-fed cropping system and maize is the main crop of the region. Basic health facilities are mainly provided by few public health dispensaries in the region, but locals residing at higher altitudes have very limited access to them and are mainly dependent upon herbal 


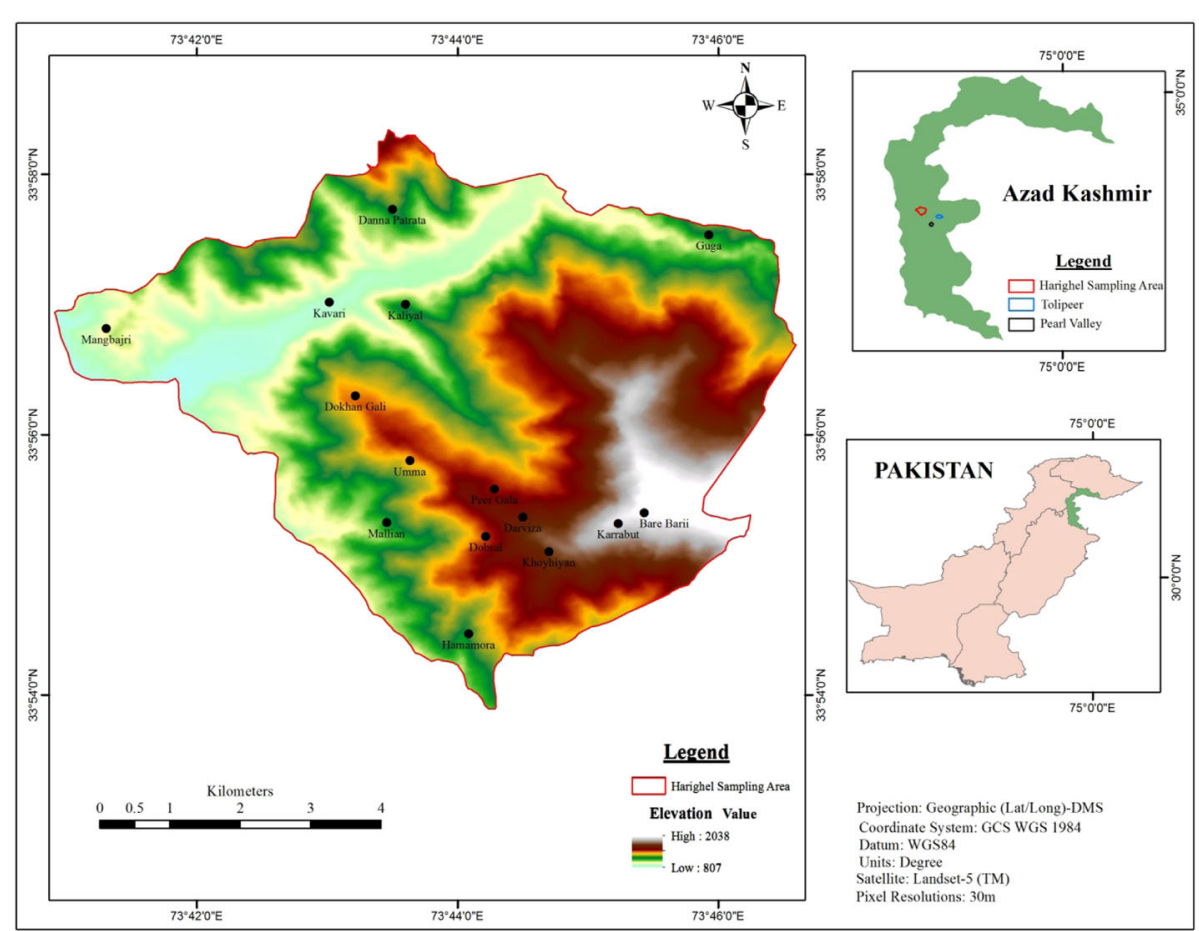

Fig. 1 Map of the study area

preparations for curing most ailments. Indigenous ethnomedicinal knowledge is mostly in the hand of elder people and health practitioners.

\section{Data collection}

Ethnomedicinal data was collected from 79 informants including 45 men and 34 women during April 2017 to
March 2018, using semistructured interviews and group discussions, after obtaining prior informed consent from the participants. Informants were selected randomly by convenience sampling (i.e., a sampling method in which units are selected based on easy access or availability) [47] and sometimes by using a snowball method [14]. Questionnaires were prepared according to Edward et al.

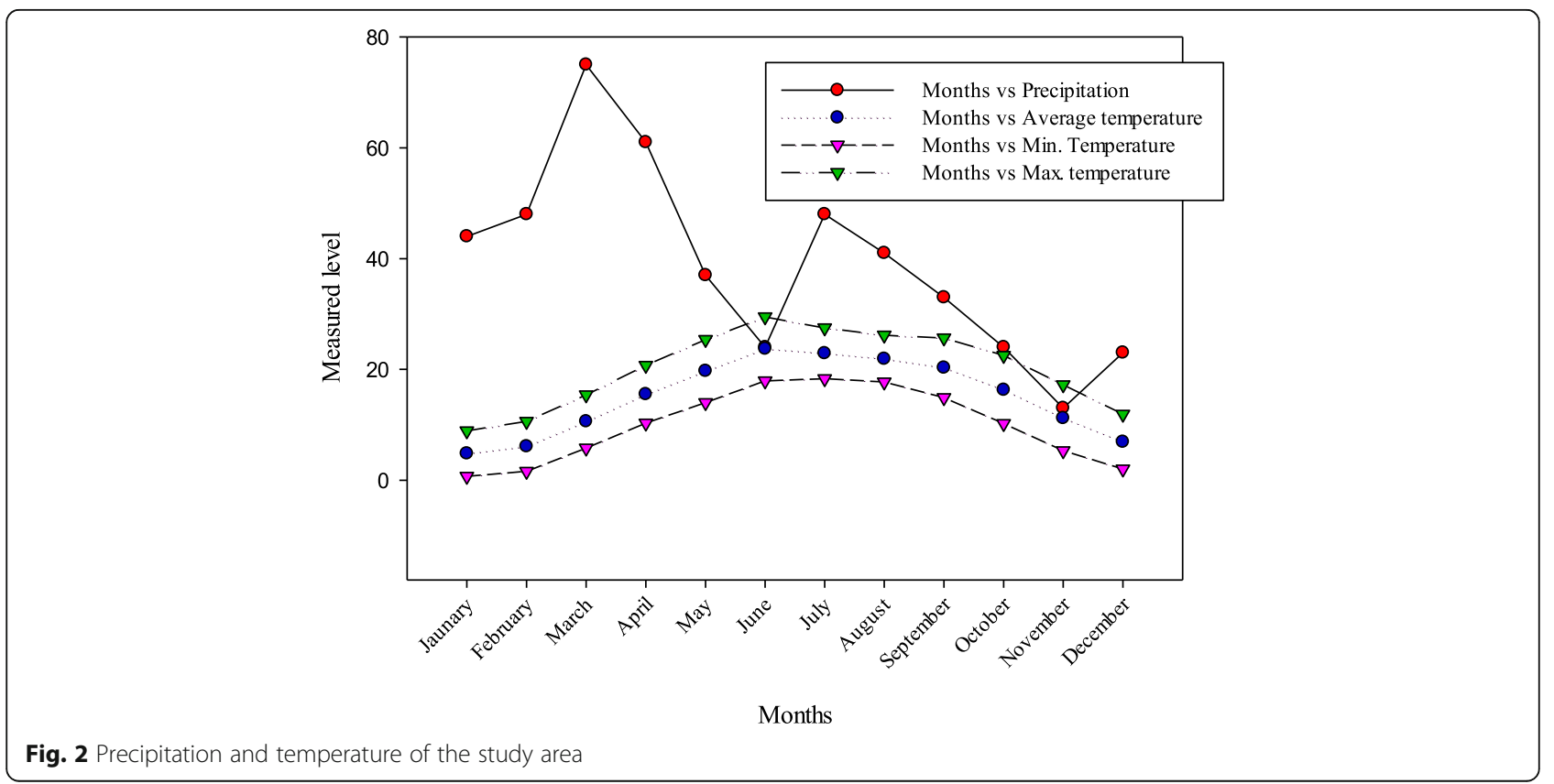


[48]. The ethical guidelines provided by International Society of Ethnobiology (http://www.ethnobiology.net/) were strictly followed. The ethical approval to conduct the study was given before initiating surveys from the Ethics Committee of the Women University of Azad Jammu \& Kashmir, Bagh. In addition to this, legal permission to conduct interviews was given by members of municipality committee. A prior informed consent form (PIC) was signed by all the informants after explaining the objective and consequence of study. The PIC was translated into local Pahari language. Sample size was determined by following Kadam and Bhalerao [49].

\section{Plant collection and identification}

The medicinal plants were collected dried, pressed, and mounted on standard herbarium sheets following standard taxonomic methods [50]. The specimens were identified with the help of plant taxonomist and confirmed using flora of Pakistan (https://http://www.efloras.org) [51, 52]. Further verification of identified specimens was done at the Herbarium of Medicinal and Aromatic Plants in AJ\&K established by the Pakistan Agriculture and Research Council (PARC). APG IV (2016) [53] was used for family taxonomy while The Plant List (2013) [54] was used to verify scientific names. The final voucher specimens were deposited in herbarium of the Women University of AJ\&K, Bagh.

\section{Ethnobotanical indices}

The homogeneity and validation of collected ethnomedicinal data was checked by applying following quantitative indices.

\section{Informant consensus factor}

Emic use types were grouped in to 16 etic use categories following International Classification of Primary Care (ICPC) with some modification [55]. The agreement between the respondents about usage of plants for curing various groups of ailments was checked by informant consent factor. It was calculated by following Heinrich et al. [56] using given formula:

$$
F i c=\frac{N u r-N t}{(\text { Nur }-1)}
$$

Where;

Nur $=$ use-reports in selected group of diseases, $\mathrm{Nt}=$ species used for treating various diseases of that group. Informant consensus factor (ICF) values varied from 0 to 1 , where value (close to 1 ) indicates that plant species are selected by using well-defined criteria or information and its uses are extremely exchanged among the informants and low values (close to 0) are obtained when plant species are chosen randomly or information about their use are not exchange among informants [20].

\section{Relative frequency of citation}

The harmony between respondents on medicinal uses of plants in the study area was determined by relative frequency of citation (RFC). It was calculated by following Vijayakumar et al. [57] using the given formula:

$$
R F C=F C / N
$$

Where;

$\mathrm{FC}=$ informants reporting use of a given species, $N=$ total number of informants. This index is used to identify the most utilized/preferred plants in the area. FC value varies from 0 (when a plant species is not under any use in that area) to 1 (if all the informants refer plant species as useful). FC exhibits the local importance of each species without considering the use categories [31, 58].

\section{Use value index}

The use value of plant species was determined by following Vijayakumar et al. [57] using the given formula:

$$
\mathrm{UV}=\frac{\Sigma \mathrm{Ui}}{\mathrm{N}}
$$

\section{Where;}

$\mathrm{Ui}=$ use reports cited by each respondent for given species, $N=$ total number of respondents. Use value reflects the relative importance of reported plant species in area. High use value shows that plant species have many use reports and is important in the region, whereas low use value (approach to 0) shows that species have few use reports related to its use. However, use report is not meaningful to differentiate whether a plant species is used for single or manifold purposes [20].

\section{Relative importance}

It was calculated following Khan et al. [6] by using the given formula.

$$
R I=(\operatorname{RelPH}+\operatorname{RelBS}) \times 100 / 2
$$

$\mathrm{PH}=$ pharmacological attribute of the selected plant, Rel $\mathrm{PH}=$ relative pharmacological attributes of a given plant.

$$
\text { RelPH }=\frac{\mathrm{PH} \text { of a selected plant }}{\text { maximum PH of all plant species }}
$$

BS = body systems treated by selected plant species, Rel $\mathrm{BS}=$ relative body systems treated by selected species. 


$$
\text { RelBS }=\frac{\mathrm{BS} \text { of a given plant }}{\text { maximum BS of all reported plant species }}
$$

\section{Fidelity level}

Fidelity level indicates the preference of particular plant species by informants to treat specific disease. It was calculated following Alexiades and Sheldon, [59] by using the given formula

$$
F L \%=N p / N \times 100
$$

Where;

$\mathrm{Np}=$ informants reporting use of particular plant species for a specific disease category, $N=$ total number of informants who mentioned uses for a specific plant species for all disease category. High fidelity level (FL) value shows maximum frequency of use by the informants to treat a particular disease [20].

\section{Jaccard index}

The similarity of knowledge between different communities was determined by comparing the findings of the current study with 22 published peer reviewed papers at regional, national, and global level by applying Jaccard index. These includes 9 studies from Azad Jammu \& Kashmir, 7 form Khyber Paktunkhawa, and 3 from other areas of Pakistan. The studies conducted on the areas with similar, vegetation, climatic condition, and culture were consider for comparison. Further, the findings were also compared with 3 studies conducted in other developing counties including Nepal, India, and Ethopia. Jaccard index (JI) was calculated following Gonza et al. [60] by using the given formula:

$$
\mathrm{JI}=\frac{\mathrm{c} \times 100}{(\mathrm{a}+\mathrm{b})-\mathrm{c}}
$$

Where;

$a=$ species of the study area, $b=$ species of the neighboring area, and $c=$ number of species common to both area.

\section{Results and discussion}

\section{Demography and knowledge variation}

A total of 79 informants were interviewed to collect medicinal plant knowledge based on their gender, age, and education (Table 1). The first category used for classification of informants was gender and 45 men and 34 women were interviewed. The easier availability and approachability to male informants and the prohibition of interaction of women with strangers, as well as and veiling (parda) forced us to interview more men than women. Demographic data demonstrates that women

\begin{tabular}{|c|c|c|c|c|}
\hline Variables & IC & Number & ANSRI & ANURI \\
\hline \multirow[t]{3}{*}{ Gender } & Men & 45 & 4.98 & 8.05 \\
\hline & Women & 34 & 5.72 & 9.38 \\
\hline & Total & 79 & & \\
\hline \multirow[t]{3}{*}{ Age group } & $20-40$ & 22 & 3.96 & 3.10 \\
\hline & $41-60$ & 46 & 8.40 & 4.96 \\
\hline & $60-80$ & 11 & 12.70 & 11.35 \\
\hline \multirow[t]{6}{*}{ Education Level } & Illiterate & 26 & 5.95 & 4.08 \\
\hline & Elementary education & 18 & 12.25 & 6.70 \\
\hline & Secondary education & 13 & 11.90 & 6.11 \\
\hline & HSE & 10 & 6.60 & 5.55 \\
\hline & Bachelor degree & 7 & 6.15 & 5.01 \\
\hline & Higher education & 5 & 10.80 & 6.71 \\
\hline \multirow[t]{7}{*}{ Professions } & THPs & 13 & 25.55 & 13.64 \\
\hline & Midwives & 10 & 13.2 & 10.43 \\
\hline & Herders & 06 & 9.10 & 8.12 \\
\hline & Housewives & 18 & 6.85 & 6.15 \\
\hline & Farmers & 08 & 5.25 & 4.45 \\
\hline & Teachers & 10 & 6.71 & 7.10 \\
\hline & Others & 14 & 4.55 & 3.93 \\
\hline
\end{tabular}
(average known species $=5.72$; average cited uses $=$
Table 1 Demographic information of the Informants

IC informants category, ANSRI average number of species reported by each informant, ANURI average number of use reported by each informant, HSE higher secondary education, THPs traditional health practionaires

9.38) had more knowledge about plants than men (average known species $=4.98$; average cited uses $=8.05$ ) . Division of labor between genders in the area may be one reason for this difference, as men generally manage the fieldwork and earning, while women manage the indoor activities and domestic life, which are highly associated with herbal preparations to keep the family healthy. Similar findings were reported by other studies including Qaseem et al. [40] from Kotli, Ahmad et al. [44] from Neelum valley, and Kyani et al. [20] from Abbottabad. Age was used as second classification criterion and informants were classified into three major categories, i.e., above 60, between 40 and 60 and less than 40. Elders (age above 60) had more knowledge about plants than young people (age less than 40). Another reason for lower knowledge of young informants was their limited interest in herbal preparations due to changes in lifestyle with advent of industrialization and modernization. These findings were supported by other reports including Qaseem et al. [40] from Kotli and Umair et al. [61] from Hafizabad. Education was a third influential factor. Uneducated informants had a vast ethnobotanical knowledge, while tan educated informants had a more limited knowledge of plants. Likewise, traditional health practitioners had a broad traditional knowledge about medicinal uses of plants compared to other professions. 
Highly educated informants usually relied on allopathic medicines for their immediate healthcare, and had least knowledge about herbal medicines and their preparation methods. These finding are supported nationally by Kayani et al. [20], Yaseen et al. [22], and internationally by Giday et al. [62] and Tugume et al. [63].

\section{Diversity of ethnomedicinal flora}

A total of 150 medicinal plants belonging to 60 families and 98 genera were reported from study area (Table 2). Out of total 150 species, 76 were endemic or native and 74 exotic and among them, 136 plant species were wild, 10 were cultivated, and 4 were both wild-collected and cultivated (Table 1S). The herbaceous life form was dominantly (78 sp.; 52\%) used in herbal preparation followed by shrubs (27 sp.; 18\%), trees (25 sp.; $16.6 \%$ ), grasses (12 sp.; 8\%), ferns (5 sp.; 3.3\%), and epiphytes or climbers (Fig. 3). These findings are in accordance with previous reports $[40,41,79,80]$. The predominance of the herbaceous habit in mountainous areas is a common ecological phenomena throughout the world [17, 44, 81]. The reason might be the high rainfall and moisture content at higher altitudinal areas [20,41, 47].

Asteraceae, Fabaceae, and Rosaceae were the dominant families having 15 species each, followed by Lamiaceae and Poaceae having 12 species each (Fig. 4). Our results are in accordance with Amjad et al. [41], Kayani et al. [20], and Tariq et al. [21]. The prevalence of these families might be due to their abundance and easy accessibility in the study area. Moreover, majority of the reported species of these families possess significant pharmaceutical, pharmacological and organoleptic properties [82-85].

\section{Plant part(s) used}

Herbal recipes were prepared using different plant parts in investigated area. As shown in Table 2, leaves, whole plant, and roots were the most preferred plant parts used in herbal preparations (30.2, 16.6, and $14.4 \%$, respectively). These findings were similar as reported earlier from Pakistan and other countries [40, 44, 86-89]. Local preference of leaves in herbal recipes is because of their collection and availability. Leaves, whole plant, and roots are rich in health beneficial secondary metabolites that contribute significantly in the prevention and treatment of various health disorders [6, 44, 90-92]. Though, local inhabitants prefer to use whole plant and roots but their use is not recommended, as exploitation of whole plant species or their uprooting could cause harmful effect on regeneration and may cause species extension [93, 94].

\section{Method of preparation and administration}

Different recipes were prepared from medicinal plants by using different methods based on the actual site and type of disease treated. Decoction was the most common method (41 sp.; 17\%) for preparation of herbal recipes, followed by paste (36 sp.; 15\%), powder (30 sp.; 13\%), extract (28 sp.; 12\%), and juice (30 pp.; 13\%) (Fig. 5). Our findings are supported by previous documentation $[17,40,41,80,95,96]$. Ease of preparation might be a reason for the extensive use of decoctions to treat aliments, as it can be prepared by mixing specific part of plants with soup, tea, water, honey, milk, and butter [97]. The availability of active metabolic compounds might increase due to the fact of heating which speed up the biological reactions [98-101]. Sometimes, whole plants were used in herbal preparations. Most of the herbal recipes were prepared using single plant species assuming nontoxicity, palatability, and high efficacy. Some recipes were based on application of two or more plants to attain maximum therapeutic effects. The amount of medicinal plants and frequency of dose are based on patient condition, health, age, and disease severity. In the study area, constipation was commonly treated using the fruits of Ficus carica, and for adults 4-5 fruits were used, while 2-3 were regarded as effective for children. The frequent mode of application was internal $(76.2 \%)$ and only few preparations were applied topically (Table 2), as paste, body wash, or rubbed on the affected body parts. These findings were comparable to previous reports $[20,40,44]$.

\section{Informant consensus factor}

A total 127 emic use reports were categorized in to 16 etic use categories (Table 2S). The informant consensus factor was analyzed based on disease categories, as a single plant might be used to treat 3-4 or more disease categories. A high informant consensus factor (ICF) value reflects high dependence of local inhabitants on medicinal plants [102] and low ICF values indicate less consistency of informant's knowledge. Ailments were classified in to 18 categories to develop informant consensus. The ICF value ranged from 0.23 to 0.95 . The maximum value of ICF was estimated for digestive disease category (0.95) followed by skin problems and respiratory disorders (Fig. 6). This was strongly supported by Qaseem et al. [40], Umari et al. [78], Ullah et al. [42], Amjad et al. [41], Ahmad et al. [44], and Bib et al. [17] who also reported the maximum ICF for digestive diseases in their investigated area. ICF values are generally influenced by the number of informants and are more significant when calculated for uses cited by many informants. In general, ICF values were high in our study, revealing that the informants tend to agree on which 
Table 2 Medicinal uses of the reported taxa and their comparison with previous reports

\begin{tabular}{|c|c|c|c|c|c|}
\hline $\begin{array}{l}\text { Scientific name/ } \\
\text { voucher number/ } \\
\text { habit }\end{array}$ & Local name & $\begin{array}{l}\text { Part } \\
\text { used }\end{array}$ & $\begin{array}{l}\text { Method of } \\
\text { preparation/ } \\
\text { mode of } \\
\text { application }\end{array}$ & Diseases treated & Previous use reports \\
\hline
\end{tabular}

Acanthaceae

\section{Acanthaceae}

Dicliptera bupleuroides Somni

Nees in Wall./UZ-02/H

Lf pas, ext dec, int

Wounds

Justicia adhatoda L./ Baikher UZ-31/S

$\begin{array}{ll}\text { Bk } & \text { pow, int } \\ \text { Lf } & \text { pow, int } \\ \text { Rt } & \text { pow, int }\end{array}$

Amaranthaceae

Achyranthes aspera L./ Puthcanda UZ-90/ H

\section{Wp pas, ext}

Lf jui, int

Rt dec, int

Alternanthera pungens Khaki buti Lf dec, int

Kunth/UZ-79/H

$\begin{array}{llll}\begin{array}{lll}\text { Amaranthus spinosus } \\ \text { L./UZ-71/H }\end{array} & \begin{array}{l}\text { Jungli } \\ \text { Ganayar }\end{array} & \begin{array}{l}\text { Lf } \\ \text { Lf }\end{array} & \text { dec, int } \\ & & & \\ \text { Amaranthus viridis L./ } & \text { Ganyar } & \text { Wp } & \text { pow, int } \\ \text { UZ-50/H } & & \text { Lf } & \text { Pas, ext }\end{array}$

Amaryllidaceae

Allium griffithianum Boiss./UZ-42/H

Piazi

Ae

coo, int

Dyspepsia, Flatulence

Scorpion stings and Snake bites

\section{Eye diseases}

Inflammation

Skin Infection, Cuts and external injury

Biliousness, Eye infection

\section{Constipation}

Diarrhea, Malaria, Jaundice

Antidote against snake and spider bites

Skin diseases, Indigestion

$\begin{array}{llll}\text { Torilis japonica } & \text { Lahndara } & \text { Sd } & \text { pow, int } \\ \text { (Houtt.) DC./UZ-69/H } & & \text { Rt } & \text { dec, int }\end{array}$

Apocynaceae

Carissa opaca Stapf. ex. Haines./UZ-110/S

Garanda Wp pow, int

Joint pain, Scabies, Jaundice, Inflammation

Nerium oleander L./ Kanair

UZ-122/S

Fl jui, int

Lf jui, int

Araliaceae

Hedera nepalensis $\mathrm{K}$. Koch./UZ-147/H

Bail Lf dec, int

Asclepiadaceae

Vincetoxicum hirundinaria Medik./ Medhshingi Ae dec, int

Boils, Pimples

Nervous system disorders, Rheumatism, Abnormal sweating

Cough

Flu, Fever, Toothache, Blood pressure, Antidote

UZ-131/H

Aspleniaceae

Asplenium dalhousiae Hook./UZ-108/F
Gutti Wp dec, int

Typhoid
$10,20,3 \odot, 4 \odot, 50,60,70,80,90$, $100,110,120,130,140,150,160$, $17 \nabla, 180,190,200,21 \diamond, 22 \diamond$

$10,20,30,4 \odot, 50,60,70,80,90$, $100,110,120,130,140,150,160$, $170,180,190,200,210,220$

$1,2,3,4,5,6 \mathbf{\Lambda}, 70,8 \mathbf{\Lambda}, 90,100$, $110,120,130,140,150,160,170$, $18 \odot, 190,20 \diamond, 21 \boldsymbol{\Lambda}, 220$

$10,20,30,40,50,60,70,80,90$, $100,110,120,130,140,150,160$, $170,180,190,200,210,22 \diamond$

$10,2 \boldsymbol{\Lambda}, 30,40,50,6 \mathbf{\Lambda}, 70,80,90$, $100,110,120,130,140,150,160$, $170,180,190,200,21 \boldsymbol{\Lambda}, 220$

$1 \mathbf{\Lambda}, 2 \mathbf{\Lambda}, 30,40,50,6 \mathbf{\Lambda}, 70,8 \mathbf{\Lambda}$, $90,100,110,120,13 \mathbf{\Lambda}, 140,150$, $160,170,18 \boldsymbol{\Lambda}, 190,200,21 \mathbf{\Lambda}$,

\section{$22 \boldsymbol{\Delta}$}

$10,20,30,40,50,60,70,80,90$, $100,110,120,130,140,150,160$, $170,180,190,200,210,22 \diamond$

$10,20,30,40,5 \mathbf{\Lambda}, 60,70,80,90$, $100,110,120,13 \boldsymbol{\Lambda}, 140,150,160$, $17 \diamond, 18 \diamond, 19 \diamond, 20 \diamond, 21 \diamond, 22 \diamond$

$1 \diamond, 2 \diamond, 3 \diamond, 4 \diamond, 5 \diamond, 60,7 \diamond, 80,90,10 \diamond$, $110,120,130,140,150,160,170,180$, $19 \diamond, 200,21 \diamond, 220$

$10,20,30,4 \odot, 50,6 \boldsymbol{\Lambda}, 70,80,90$, $100,110,120,130,140,150,160$, $170,18 \odot, 190,200,210,220$

$10,20,3 \boldsymbol{\Lambda}, 4 \boldsymbol{\Lambda}, 50,6 \boldsymbol{\Lambda}, 70,80,90$ $100,110,120,130,140,150,160,170$, $180,19 \boldsymbol{\Lambda}, 200,21 \boldsymbol{\Lambda}, 22 \diamond$

$10,2 \mathbf{\Lambda}, 3 \mathbf{\Lambda}, 4 \mathbf{\Lambda}, 50,60,7 \mathbf{\Lambda}, 80$, $90,100,11 \mathbf{\Lambda}, 12 \nabla_{11}, 130,140,150,160$, $17 \diamond, 18 \diamond, 190,200,21 \diamond, 22 \diamond$

$1 \diamond, 20,3 \odot, 4 \odot, 5 \diamond, 6 \diamond, 7 \diamond, 80,90,100$ $110,120,130,140,150,160,170,180$ $19 \diamond, 200,21 \diamond, 22 \diamond$

$10,20,30,40,50,60,70,80,90,100$, $110,120,130,140,150,160,170,180$, $19 \diamond, 20 \diamond, 21 \diamond, 22 \diamond$ 
Table 2 Medicinal uses of the reported taxa and their comparison with previous reports (Continued)

\begin{tabular}{|c|c|c|c|c|c|}
\hline $\begin{array}{l}\text { Scientific name/ } \\
\text { voucher number/ } \\
\text { habit }\end{array}$ & Local name & $\begin{array}{l}\text { Part } \\
\text { used }\end{array}$ & $\begin{array}{l}\text { Method of } \\
\text { preparation/ } \\
\text { mode of } \\
\text { application }\end{array}$ & Diseases treated & Previous use reports \\
\hline
\end{tabular}

Asteraceae

Achillea millefolium L./ kangi.i $\quad \mathrm{Fl}$ pas, int

UZ-117/H

Lf dec, int

Anaphalis adnata

Wall. ex DC NZ-111/

Dialect Lf pow, ext

$\mathrm{H}$

Artemisia vulgaris L./

UZ-20/H

Chaow Lf jui, int

Bidens biternata
(Lour.) Merr. \& Sherff./

Palouthi Lf jui, int

$\mathrm{UZ}-74 / \mathrm{H}$

Cirsium vulgare (Savi) Kandiara

Ten. /UZ-32/H

Conyza Canadensis
(L.) Cronquist/UZ-01

$\mathrm{H}$

Crepis multicaulis

Ledeb./UZ-11/H

Gerbera gossypina

(Royle) Beauverd/UZ-

129/H

Helianthus annuus L./ Souraj mukhi UZ-104/H

Launaea procumbens

(Roxb.) Ram. \& Raj./

Hund

$\mathrm{UZ}-41 / \mathrm{H}$

Silybum marianum (L.) Kandiyar

Gaertn./UZ-52/H

Sonchus oleraceus (L.) Dod

L./ UZ-62/H

Tagetes minuta L./UZ- Sadberga

150/H

Taraxacum officinale

(L.) Weber ex

F.H.Wigg./UZ-93/H

Xanthium strumarium L./UZ-81/S

Hend Rt jui, int

Souriyala

Lf jui, int

Fr pow, int

Asparagaceae

Polygonatum geminiflorum Decne./ Noorialam Lf inf, int UZ- 146/H

Balsaminaceae

Impatiens edgeworthii Hook.f./UZ-21/H
Batmandar/ Wp exr, int buntil Wp past, ext
Digestive problems, Brain tonic, Female organ problems

Colds and Fever

Bleeding, Wound healing

Kill worms, Skin diseases

Sore throat

Toothache

\section{Sore jaws}

Rheumatic joints

Bleeding piles

Scanty Urination, Dysentery, Diarrhea, Hemorrhages

\section{Eye infection}

Bone fractures, Wounds, Cuts, Pain, Skin diseases

\section{Skin diseases}

Scanty urination, Curing chest infections, Liver ailments

Lung ailments, Piles, Kinney problems

Diabetes, Pain

Liver problems, Scanty urination, Stomachic,

Tonic, Respiratory tract infection

\section{Abdominal pain}

\section{Earache}

Scanty urination, Liver tonic, Diabetes

Chronic mild fever

Cooling, Infections, Urinary problems

Treat pain, Fever, Inflammation, Allergy, weakness

Urinary tract infection, Fever Burns
$10,20,30,40,5 \mathbf{\Lambda}, 6 \mathbf{\Lambda}, 70,80,90$ $100,110,120,13 \mathbf{\Lambda}, 140,150,160,170$, $180,190,20 \odot, 210,220$

$10,20,30,4 \odot, 50,60,70,80,90,100$, $110,120,130,140,150,160,170,180$ $190,200,210,220$

$1 \diamond, 2 \odot, 30,40,50,60,70,80,90,10 \diamond$, $110,120,130,140,150,160,170,180$, $190,200,21 \diamond, 22 \diamond$

$10,2 \boldsymbol{\Delta}, 30,40,50,60,70,80,90,100$, $110,120,130,140,150,160,170,180$, $190,200,210,220$

$10,2 \diamond, 3 \diamond, 4 \diamond, 50,60,70,80,90,100$, $110,120,130,140,150,160,170,180$ $19 \diamond, 200,21 \diamond, 22 \diamond$

$10,20,30,4,05 \mathbf{\Lambda}, 60,70,80,90$, $100,110,120,130,140,150,160,170$, $180,190,20 \diamond, 21 \diamond, 22 \diamond$

$10,2 \diamond, 3 \diamond, 4 \diamond, 50,60,7 \diamond, 80,90,100$, $110,120,130,140,150,160170,180$ $190,200,210,220$

$10,20,3 \boldsymbol{\Lambda}, 4 \odot, 50,60,70,80,90$, $100,110,120,130,140,150,160,170$, $180,19 \diamond, 200,210,220$

$10,20,30,40,50,60,70,80,90,100$, $110,120,130,140,150,160,170,180$, $190,200,210,220$

$10,20,30,40,50,60,70,8 \boldsymbol{\Lambda}, 90$, $100,110,120,130,140,150,160,170$, $180,190,200,21 \mathbf{\Lambda}, 220$

$10,20,30,40,50,60,7 \diamond, 80,90,10\rangle$, $11 \diamond, 12 \diamond, 13 \diamond, 140,15 \diamond, 16 \mathbf{\Lambda}, 17 \diamond, 18 \diamond$, $190,200,21 \diamond, 22 \diamond$

$10,20,30,40,50,60,70,80,90,100$, $110,120,130,140,150,160,170,180$ $190,200,21 \diamond, 22 \diamond$

$10,20,30,40,50,60,70,80,90,100$, $110,120,130,140,150,160,170,18 \mathbf{\Lambda}$ $190,200,210,22 \diamond$

$1 \odot, 2 \odot, 3 \odot, 4 \odot, 5 \boldsymbol{\Lambda}, 6 \odot, 70,80,9 \boldsymbol{\Delta}$ $100,110,12 \mathbf{\Lambda}, 130,140,150,160,170$, $18 \mathbf{\Lambda}, 190,20 \boldsymbol{\Lambda}, 210,22 \mathbf{\Lambda}$

$10,2 \odot, 30,40,50,60,70,8 \mathbf{\Delta}$ $9 \mathbf{\Lambda}, 10 \mathbf{\Lambda}, 110,120,130,140,150,160$, $170,180,190,200,21 \mathbf{\Lambda}, 22 \mathbf{\Delta}$

$10,20,30,40,50,60,70,80,90,100$, $110,120,130,140,150,160,170,180$, $190,200,210,220$

$10,20,30,40,50,60,70,80,90,100$, $110,12 \mathbf{\Delta}, 130,140,150,160,170,180$ $190,200,210,22 \diamond$ 
Table 2 Medicinal uses of the reported taxa and their comparison with previous reports (Continued)

\begin{tabular}{|c|c|c|c|c|c|}
\hline $\begin{array}{l}\text { Scientific name/ } \\
\text { voucher number/ } \\
\text { habit }\end{array}$ & Local name & $\begin{array}{l}\text { Part } \\
\text { used }\end{array}$ & $\begin{array}{l}\text { Method of } \\
\text { preparation/ } \\
\text { mode of } \\
\text { application }\end{array}$ & Diseases treated & Previous use reports \\
\hline
\end{tabular}

Berberidaceae

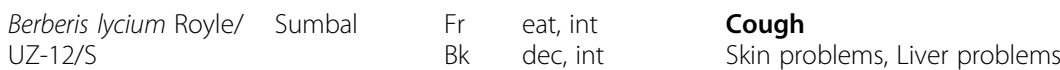

$1 \mathbf{\Lambda}, 20,3 \mathbf{\Lambda}, 4 \mathbf{\Lambda}, 5 \mathbf{\Lambda}, 6 \mathbf{\Lambda}, 7 \mathbf{\Lambda}$ $80,90,10 \boldsymbol{\Lambda}, 110,120,13 \mathbf{\Lambda}, 140,150$, $160,170,18 \boldsymbol{\Lambda}, 19 \boldsymbol{\Lambda}, 20 \boldsymbol{\Lambda}, 210,22 \boldsymbol{\Delta}$

Boraginaceae

Cynoglossum

lanceolatum Forssk./

Churoun Rt exr, int

Throat ache

UZ-140/H

Trichodesma indicum

(L.) Lehm./UZ-03/H

$\begin{array}{lll}\text { Handusibooti } & \mathrm{Fl} & \text { fra, int } \\ & \text { Lf/ } & \text { exr, int }\end{array}$

Rt

Brassicaceae

Capsella bursa-pastoris Saag

(L.) Medic./UZ-33/H

Wp eat, int

Sd pow, int

Sd flu, Int

Buxaceae

Sarcococca saligna (D. Ladan/ Lf dec, int

Don) Muel /UZ-43/H bansathra Rt jui, int

Cannabaceae

Cannabis sativa L./UZ- Bhang Lf pow, int

54/H

Caprifoliaceae

Viburnum

grandiflorum Wall. ex

Guch Sd jui, int

DC./UZ-72/S

Celastraceae

Maytenus nemorosa

Marais /UZ-82/S

Patakhi Wp ext, ext

Chenopodiaceae

Chenopodium album L./UZ-61/H

SkhaBotey Wp dec, ext

Convolvulaceae

Convolvulus arvensis L./UZ-103/C

Hirrankhuri Wp ext, int
Ipomoea purpurea (L.) Bahrwa

Roth /UZ-2/H

Wp pou, Int

Sd pow, Int

Commelinaceae

Commelina

benghalensis L./UZ-

128/S

Cornaceae

Cornus macrophylla Wall./UZ-133/T
Brain refreshment

Scanty urination, Blood purifier

Chest infections

Bleeding

Joint pain, Dysentery

Gonorrhea

Astringent that bowels stomachic, Leprosy, Tonic, Narcotic action

Typhoid, Whooping cough

Toothache, Eye inflammation

Skin diseases

Piles, Dandruff, Constipation

Skin disease, Cancer

Tonic, constipation

Skin diseases

Backache
$10,20,30,40,50,6 \mathbf{\Lambda}, 70,80,90$ $100,110,12 \mathbf{\Lambda}, 130,140,150,160,170$, $180,190,20 \boldsymbol{\Delta}, 210,220$

$10,20,30,4 \boldsymbol{\Lambda}, 5 \boldsymbol{\Lambda}, 6 \odot, 70,80,9 \boldsymbol{\Delta}$ $100,110,120,130,140,150,160,170$,

$18 \boldsymbol{\Lambda}, 190,20 \diamond, 21 \boldsymbol{\Lambda}, 22 \diamond$

$10,20,30,40,50,6 \mathbf{\Lambda}, 70,80,90$, $100,110,12 \diamond, 13 \odot, 140,150,160,17 \diamond$, $180,190,200,210,220$

$10,2 \boldsymbol{\Lambda}, 3 \odot, 4 \odot, 50,60,70,80,90$, $100,11 \diamond, 120,13 \diamond, 14 \diamond, 150,160,170$, $180,190,200,210,220$

$1 \mathbf{\Lambda}, 20,30,40,5 \boldsymbol{\Lambda}, 60,7 \mathbf{\Lambda}, 80,90$, $10 \boldsymbol{\Lambda}, 11 \mathbf{\Lambda}, 120,13 \mathbf{\Lambda}, 140,150,160$

$17 \boldsymbol{\Lambda}, 18 \boldsymbol{\Lambda}, 19 \boldsymbol{\Lambda}, 20 \boldsymbol{\Lambda}, 21 \boldsymbol{\Lambda}, 22 \boldsymbol{\Lambda}$

$\boldsymbol{\Lambda}, 20,3 \odot, 4 \odot, 50,60,70,80,90$, $10 \boldsymbol{\Lambda}, 110,120,13 \mathbf{\Lambda}, 140,150,160,170$, $180,190,200,210,220$

$10,20,30,40,50,60,70,80,90,100$, $110,120,130,140,150,160,170,180$, $190,200,210,22 \diamond$

$1 \mathbf{\Lambda}, 2 \mathbf{\Lambda}, 30,40,50,6 \mathbf{\Lambda}, 70,80,90$, $100,11 \mathbf{\Lambda}, 12 \mathbf{\Lambda}, 13 \mathbf{\Lambda}, 140,15 \mathbf{\Lambda}, 160$, $170,18 \mathbf{\Lambda}, 19 \boldsymbol{\Lambda}, 208,21 \mathbf{\Lambda}, 22 \boldsymbol{\Lambda}$

$10,20,30,40,50,60,70,8 \mathbf{\Delta}, 90$, $100,110,120,130,14 \boldsymbol{\Lambda}, 150,160,17 \boldsymbol{\Lambda}$ $180,19 \boldsymbol{\Delta}, 200,21 \mathbf{\Lambda}, 22 \diamond$

$10,2 \boldsymbol{\Lambda}, 30,40,50,60,70,80,90$, $100,110,120,130,140,150,160,170$ $180,190,200,210,22 \diamond$

$10,20,30,40,50,60,70,80,90,100$, $110,120,130,140,150,160,170,180$, $190,200,21 \diamond, 22 \diamond$

$1 \diamond, 20,30,40,50,60,70,80,90,10 \diamond$, $110,120,130,140,150,160,170,180$ $190,200,210,22 \diamond$

Cuscutaceae 
Table 2 Medicinal uses of the reported taxa and their comparison with previous reports (Continued)

\begin{tabular}{|c|c|c|c|c|c|}
\hline $\begin{array}{l}\text { Scientific name/ } \\
\text { voucher number/ } \\
\text { habit }\end{array}$ & Local name & $\begin{array}{l}\text { Part } \\
\text { used }\end{array}$ & $\begin{array}{l}\text { Method of } \\
\text { preparation/ } \\
\text { mode of } \\
\text { application }\end{array}$ & Diseases treated & Previous use reports \\
\hline $\begin{array}{l}\text { Cuscuta reflexa Roxb/ } \\
\text { UZ-12/H }\end{array}$ & Neeladari & Wp & jui, int & Jaundice, Dandruff & 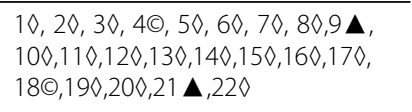 \\
\hline \multicolumn{6}{|l|}{ Cyperaceae } \\
\hline $\begin{array}{l}\text { Cyperus rotundus L./ } \\
\text { UZ- } 145 / \mathrm{H}\end{array}$ & Kah & Rt & mix, int & $\begin{array}{l}\text { Dysentery, Gastric problems, Intestinal } \\
\text { disorders }\end{array}$ & $\begin{array}{l}10,2 \mathbf{\Lambda}, 30,40,50,6 \mathbf{\Lambda}, 70,8 \mathbf{\Delta} \\
9 \mathbf{\Lambda}, 100,110,120,13 \mathbf{\Delta}, 140,150,160 \\
170,180,190,200,21 \mathbf{\Lambda}, 220\end{array}$ \\
\hline \multicolumn{6}{|l|}{ Dryopteridaceae } \\
\hline $\begin{array}{l}\text { Dryopteris filix-mas (L.) } \\
\text { Schoot/UZ-120/F }\end{array}$ & Kungi & $\mathrm{Fd}$ & veg, int & Constipation & $\begin{array}{l}10,20,30,40,50,60,70,80,90,100 \\
110,120,130,140,150,160,170,180 \\
190,200,210,220\end{array}$ \\
\hline \multicolumn{6}{|l|}{ Elaeagnaceae } \\
\hline $\begin{array}{l}\text { Elaeagnus umbellata } \\
\text { Thunb./UZ-22/S }\end{array}$ & Kankolii & $\begin{array}{l}\mathrm{Sd} \\
\mathrm{Ol} \\
\mathrm{Fl}\end{array}$ & $\begin{array}{l}\text { pow, int } \\
\text { exr, int } \\
\text { dec, int }\end{array}$ & $\begin{array}{l}\text { Stimulant in Cough } \\
\text { Pulmonary infections } \\
\text { Cardiac problems }\end{array}$ & $\begin{array}{l}1 \diamond, 2 \diamond, 3 \odot, 4 \odot, 5 \diamond, 6 \diamond, 7 \diamond, 8 \diamond, 90,10 \diamond, \\
11 \diamond, 12 \diamond, 130,14 \diamond, 150,160,17 \diamond, 180, \\
19 \diamond, 20 \diamond, 21 \diamond, 22 \diamond\end{array}$ \\
\hline \multicolumn{6}{|l|}{ Euphorbiaceae } \\
\hline $\begin{array}{l}\text { Euphorbia helioscopia } \\
\text { L./UZ-13/H }\end{array}$ & Dhoudhal & $\begin{array}{l}\text { Wp } \\
W p \\
W p\end{array}$ & $\begin{array}{l}\text { ert, ext } \\
\text { pow, int } \\
\text { lat, Int }\end{array}$ & $\begin{array}{l}\text { Kill worms } \\
\text { Healing wounds } \\
\text { Poisonous effect that cause swelling in skin }\end{array}$ & 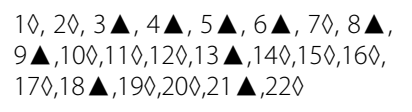 \\
\hline $\begin{array}{l}\text { Ricinus communis L./ } \\
\text { UZ-04/S }\end{array}$ & Arind & $\begin{array}{l}S d \\
L f\end{array}$ & $\begin{array}{l}\text { oil, int } \\
\text { dec, ext }\end{array}$ & $\begin{array}{l}\text { Constipation } \\
\text { Skin diseases, Joint pain, Muscles swelling, Eye } \\
\text { infection }\end{array}$ & $\begin{array}{l}10,2 \boldsymbol{\Delta}, 30,40,50,60,70,80,90 \\
100,110,120,130,140,150,160 \\
17 \mathbf{\Lambda}, 180,190,200,210,220\end{array}$ \\
\hline \multicolumn{6}{|l|}{ Fabaceae } \\
\hline $\begin{array}{l}\text { Acacia modesta Wall./ } \\
\text { UZ-63/T }\end{array}$ & Kikar & $\mathrm{Br}$ & rfo, int & Anti-inflammatory, Toothache & $\begin{array}{l}1 \diamond, 2 \diamond, 3 \diamond, 40,50,6 \mathbf{\Delta}, 7 \diamond, 8 \diamond, 9 \diamond \\
100,110,120,130,140,150,160,17 \mathbf{\Lambda} \\
180,19 \mathbf{\Delta}, 200,21 \mathbf{\Delta}, 22 \diamond\end{array}$ \\
\hline $\begin{array}{l}\text { Acacia nilotica (L.) } \\
\text { Delile/UZ-84/T }\end{array}$ & Desi kikar & $\begin{array}{l}\mathrm{Bk} \\
\mathrm{Pd}\end{array}$ & $\begin{array}{l}\text { pas, int } \\
\text { pow, ext }\end{array}$ & $\begin{array}{l}\text { Piles, Dysentery, Diarrhea } \\
\text { Anti-dandruff }\end{array}$ & 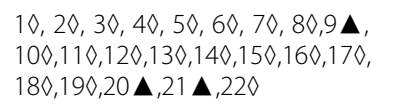 \\
\hline $\begin{array}{l}\text { Astragalus canadensis } \\
\text { L./UZ-125/H }\end{array}$ & Tindni & Lf & inf, int & Stomachache & $\begin{array}{l}1 \diamond, 20,30,40,50,60,70,8090,100 \\
11 \diamond, 120,130,140,150,160,170,180 \\
19 \diamond, 200,21 \diamond, 22 \diamond\end{array}$ \\
\hline $\begin{array}{l}\text { Bauhinia variegata L./ } \\
\text { UZ-14/T }\end{array}$ & Kachnar & $\mathrm{Fl}$ & inf, int & Tonic, Gastric problems & $\begin{array}{l}10,20,30,40,50,60,70,80,90,100 \\
110,120,130,140,150,160,170,180 \\
190,200,21 \diamond, 220\end{array}$ \\
\hline $\begin{array}{l}\text { Crotalaria juncea L./ } \\
\text { UZ-95/H }\end{array}$ & Sunn & $\begin{array}{l}\text { Sd } \\
\text { Rt }\end{array}$ & $\begin{array}{l}\text { pas, ext } \\
\text { exr, int }\end{array}$ & $\begin{array}{l}\text { Hair tonic, Skin diseases } \\
\text { Colic, Epistaxis }\end{array}$ & $\begin{array}{l}1 \diamond, 20,30,40,50,60,70,80,90,100 \\
11 \diamond, 120,130,140,150,160,170,180 \\
19 \diamond, 200,21 \diamond, 220\end{array}$ \\
\hline $\begin{array}{l}\text { Dalbergia sissoo DC./ } \\
\text { UZ- } 55 / T\end{array}$ & Shishm & Lf & dec, int & $\begin{array}{l}\text { Eye pain, Body pain, Diarrhea, Jaundice, } \\
\text { Abdominal pain, Skin diseases }\end{array}$ & $\begin{array}{l}1 \diamond, 20,30,40,50,60,70,80,90,100, \\
11 \diamond, 120,130,140,150,160,170,18 \mathbf{\Delta}, \\
19 \diamond, 200,21 \mathbf{\Lambda}, 22 \diamond\end{array}$ \\
\hline $\begin{array}{l}\text { Desmodium elegans } \\
\text { DC./UZ-142/S }\end{array}$ & Halphaat & Rt & tea, int & Hypertension, Antidote & $\begin{array}{l}1 \diamond, 2 \boldsymbol{\Delta}, 30,40,50,60,70,80,90 \\
10 \diamond, 110,12 \diamond, 130,140,150,160,17 \diamond \\
18 \diamond, 19 \diamond, 20 \diamond, 21 \diamond, 22 \diamond\end{array}$ \\
\hline $\begin{array}{l}\text { Indigofera linifolia (L. } \\
\text { f.) Retz./UZ-46/H }\end{array}$ & Jund & Lf & rfo, ext & Skin allergy & $\begin{array}{l}10,20,30,40,50,6,70,80,90,100 \\
110,120,130,140,150,160,170,180 \\
190,200,210,22 \diamond\end{array}$ \\
\hline $\begin{array}{l}\text { Lathyrus aphaca L./ } \\
\text { UZ-136/H }\end{array}$ & Jangli matter & $\mathrm{Sd}$ & pow, int & Narcotic & 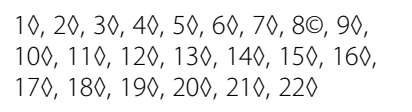 \\
\hline $\begin{array}{l}\text { Lespedeza juncea (L.f.) } \\
\text { Pers./UZ-78/H }\end{array}$ & Kuchani & Rt & jui, int & Diarrhea, Dysentery & $\begin{array}{l}1 \diamond, 2 \mathbf{\Delta}, 3 \odot, 4 \odot, 5 \diamond, 6 \diamond, 7 \diamond, 8 \diamond, 9 \diamond \\
10 \diamond, 11 \diamond, 12 \diamond, 130,14 \diamond, 15 \diamond, 16 \diamond, 170 \\
18 \diamond, 19 \diamond, 20 \diamond, 210,22 \diamond\end{array}$ \\
\hline Lotus corniculatus L./ & Sriri & $\mathrm{Fl}$ & pou, Int & Cardiotonic, Sleeping problems & $10,20,30,40,50,60,70,80,90,100$ \\
\hline
\end{tabular}


Table 2 Medicinal uses of the reported taxa and their comparison with previous reports (Continued)

\begin{tabular}{|c|c|c|c|c|c|}
\hline $\begin{array}{l}\text { Scientific name/ } \\
\text { voucher number/ } \\
\text { habit }\end{array}$ & Local name & $\begin{array}{l}\text { Part } \\
\text { used }\end{array}$ & $\begin{array}{l}\text { Method of } \\
\text { preparation/ } \\
\text { mode of } \\
\text { application }\end{array}$ & Diseases treated & Previous use reports \\
\hline UZ-105/H & & $\begin{array}{l}\mathrm{Rt} \\
\mathrm{Wp}\end{array}$ & $\begin{array}{l}\text { exr, int } \\
\text { pou, ext }\end{array}$ & $\begin{array}{l}\text { Gastric problems } \\
\text { Tonic } \\
\text { Skin inflammation }\end{array}$ & $\begin{array}{l}110,120,130,140,150,160,170,180 \\
19 \diamond, 200,21 \diamond, 22 \diamond\end{array}$ \\
\hline $\begin{array}{l}\text { Medicago polymorpha } \\
\text { L./UZ-66/H }\end{array}$ & Maina & Lf & exr, int & Dysentery, Indigestion & $\begin{array}{l}10,20,30,40,50,60,70,80,90,100 \\
110,120,130,140,150,160,170,18 \mathbf{\Delta} \\
19 \boldsymbol{\Lambda}, 200,210,22 \diamond\end{array}$ \\
\hline $\begin{array}{l}\text { Mimosa pudica L./UZ- } \\
\text { 118/H }\end{array}$ & Choi Moi & Lf & dec, int & $\begin{array}{l}\text { Hemorrhoids, Urinary infections, Cancer, } \\
\text { Diabetes, , Hepatitis, Obesity, Sores, Piles, } \\
\text { Glandular swellings }\end{array}$ & $\begin{array}{l}1 \diamond, 20,30,4 \diamond, 5 \diamond, 6 \diamond, 7 \diamond, 80,90,10 \diamond \\
11 \diamond, 120,130,140,150,160,170,18 \diamond \\
190,200,21 \diamond, 220\end{array}$ \\
\hline $\begin{array}{l}\text { Trifolium resupinatum } \\
\text { L./UZ-85/H }\end{array}$ & Tilpetra & Sd & che, int & Digestive disorder like Abdominal pain. & 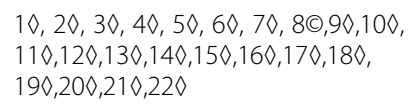 \\
\hline $\begin{array}{l}\text { Vicia sativa L./UZ-25/ } \\
\text { C }\end{array}$ & Phalli & Wp & inf, int & $\begin{array}{l}\text { Indigestion, Tonic, Scanty urination, Asthma, } \\
\text { Cough, Bronchitis, Skin diseases, Urinary } \\
\text { problems }\end{array}$ & $\begin{array}{l}1 \diamond, 20,30,40,50,6 \mathbf{\Lambda}, 70,80,90 \\
10 \diamond, 110,120,130,140,150,160,170 \\
180,190,200,210,220\end{array}$ \\
\hline \multicolumn{6}{|l|}{ Geraniaceae } \\
\hline $\begin{array}{l}\text { Geranium } \\
\text { rotundifolium LNZ- } \\
34 / \mathrm{H}\end{array}$ & Rattan joge & Rt & inf, int & Mouth ulcers, Stomach ulcer, Hemorrhoids & $\begin{array}{l}1 \diamond, 20,30,4 \diamond, 5 \diamond, 6 \diamond, 7 \diamond, 80,90,10 \diamond \\
11 \diamond, 120,130,14 \diamond, 150,160,17 \diamond, 18 \diamond \\
190,200,21 \diamond, 22 \diamond\end{array}$ \\
\hline \multicolumn{6}{|l|}{ Gentianaceae } \\
\hline $\begin{array}{l}\text { Swertia cordata (Wall. } \\
\text { ex G. Don) C.B. } \\
\text { Clarke/UZ-44/H }\end{array}$ & Cheratbotay & $\mathrm{Ae}$ & exr, int & $\begin{array}{l}\text { Digestive problem, Liver problems, Diabetes, } \\
\text { Nausea }\end{array}$ & $\begin{array}{l}1 \diamond, 2 \boldsymbol{\Delta}, 30,40,50,60,70,80,90 \\
100,110,120,130,140,150,160,17 \diamond \\
18 \diamond, 19 \diamond, 200,21 \diamond, 22 \diamond\end{array}$ \\
\hline \multicolumn{6}{|l|}{ Juglandaceae } \\
\hline $\begin{array}{l}\text { Juglans regia L./UZ- } \\
53 / T\end{array}$ & Akhrot & $\begin{array}{l}\mathrm{Ft} \\
\mathrm{Lf} / \\
\mathrm{Bk}\end{array}$ & $\begin{array}{l}\text { rfo, int } \\
\text { rfo, ext }\end{array}$ & $\begin{array}{l}\text { Brain and physical weakness } \\
\text { Toothache }\end{array}$ & $\begin{array}{l}1 \odot, 2 \odot, 3 \mathbf{\Delta}, 4 \mathbf{\Lambda}, 50,60,70,80,9 \diamond \\
10 \mathbf{\Delta}, 110,120,130,140,150,16 \diamond, 17 \diamond \\
18 \mathbf{\Lambda}, 19 \mathbf{\Delta}, 20 \mathbf{\Delta}, 210,22 \mathbf{\Delta}\end{array}$ \\
\hline \multicolumn{6}{|l|}{ Lamiaceae } \\
\hline $\begin{array}{l}\text { Ajuga bracteosa Wall. } \\
\text { ex Benth./UZ-94/H }\end{array}$ & Rattibotti & $\begin{array}{l}\text { Ae } \\
\text { Lf }\end{array}$ & $\begin{array}{l}\text { exr, int } \\
\text { exr, int }\end{array}$ & $\begin{array}{l}\text { Blood purifier, Pimples } \\
\text { Inflammation, Earache, pain }\end{array}$ & $\begin{array}{l}1 \diamond, 20,3 \odot, 4 \odot, 5,6 \mathbf{\Lambda}, 70,80,9 \diamond, 10 \diamond, \\
110,12 \diamond, 13 \odot, 140,150,160,17 \mathbf{\Lambda}, 180, \\
190,20 \mathbf{\Delta}, 21 \diamond, 220\end{array}$ \\
\hline $\begin{array}{l}\text { Callicarpa macrophylla } \\
\text { Vahl/UZ-77/S }\end{array}$ & Bengli & $\begin{array}{l}\text { Lf } \\
\text { Rt } \\
\text { Bk }\end{array}$ & $\begin{array}{l}\text { dec, ext } \\
\text { inf, int } \\
\text { pas, ext }\end{array}$ & $\begin{array}{l}\text { Alleviating pain in rheumatism, Diarrhea, } \\
\text { Dysentery } \\
\text { Relieving rashes of tongue } \\
\text { Wounds and cuts }\end{array}$ & $\begin{array}{l}1 \diamond, 2 \diamond, 3 \diamond, 4 \diamond, 5 \diamond, 6 \diamond, 7 \diamond, 8 \diamond, 9 \diamond, 10 \diamond \\
11 \diamond, 12 \diamond, 13 \diamond, 14 \diamond, 150,16 \diamond, 17 \diamond, 18 \diamond \\
19 \diamond, 200,21 \diamond, 22 \diamond\end{array}$ \\
\hline $\begin{array}{l}\text { Mentha spicata L./UZ- } \\
132 / \mathrm{H}\end{array}$ & Bebrii & Lf & pas, int & Cooling agent, Gastric problem & $\begin{array}{l}1 \diamond, 2 \diamond, 3 \diamond, 4 \diamond, 5 \diamond, 6 \diamond, 7 \diamond, 8 \diamond, 9 \diamond, 10 \diamond \\
11, \mathbf{\Lambda}, 120,13 \diamond, 14 \diamond, 15 \diamond, 16 \diamond, 170,18 \diamond \\
19 \odot, 20 \diamond, 21 \diamond, 22 \diamond\end{array}$ \\
\hline $\begin{array}{l}\text { Mentha longifolia (L.) } \\
\text { L./UZ-83/H }\end{array}$ & Podina & $\begin{array}{l}\text { Wp } \\
\text { Lf }\end{array}$ & $\begin{array}{l}\text { dec, int } \\
\text { pas, Int }\end{array}$ & $\begin{array}{l}\text { Stimulant, } \\
\text { Cough, Flatulence, Digestive disorders }\end{array}$ & $\begin{array}{l}1 \diamond, 20,30,40,50,6 \diamond, 7 \diamond, 80,90,10 \diamond \\
110,120,130,140,150,160,170,180 \\
190,200,21 \diamond, 22 \diamond\end{array}$ \\
\hline $\begin{array}{l}\text { Micromeria biflora } \\
\text { (Buch.-Ham. ex } \\
\text { D.Don) Benth/UZ-76/ } \\
\text { S }\end{array}$ & Shahibooti & $\begin{array}{l}\text { Lf } \\
\text { Lf }\end{array}$ & $\begin{array}{l}\text { oil, int } \\
\text { jui, int }\end{array}$ & $\begin{array}{l}\text { Headache } \\
\text { Digestive disorders }\end{array}$ & $\begin{array}{l}1 \diamond, 2 \mathbf{\Delta}, 3 \diamond, 4 \diamond, 5 \odot, 6 \mathbf{\Delta}, 7 \diamond, 8 \diamond, 9 \diamond \\
10 \diamond, 11 \diamond, 12 \diamond, 13 \diamond, 14 \diamond, 15 \diamond, 16 \diamond, 17 \diamond \\
18 \diamond, 19 \diamond, 20 \diamond, 21 \diamond, 22\end{array}$ \\
\hline $\begin{array}{l}\text { Ocimum sanctum L/. } \\
\text { UZ-134/H }\end{array}$ & Bebrii & Lf & pas, int & Cooling agent, Gastric problem & 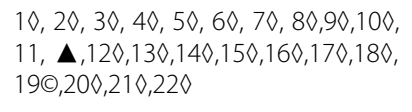 \\
\hline $\begin{array}{l}\text { Origanum vulgare L./ } \\
\text { UZ-102/H }\end{array}$ & Sahthar & Wp & pow, int & Stomach-ache, Skin infections & $\begin{array}{l}10,2 \mathbf{\Lambda}, 30,40,50,6 \mathbf{\Delta}, 70,80,90 \\
10 \mathbf{\Delta}, 110,12 \mathbf{\Delta}, 130,140,150,160,17 \diamond \\
180,190,20 \diamond, 21 \diamond, 220\end{array}$ \\
\hline $\begin{array}{l}\text { Otostegia limbata } \\
\text { (Benth.) Boiss./UZ-23/ } \\
\text { S }\end{array}$ & Chittipataki & $\begin{array}{l}\text { Rt } \\
\text { Rt }\end{array}$ & $\begin{array}{l}\text { ash, ext } \\
\text { dec, ext }\end{array}$ & $\begin{array}{l}\text { Wound healing } \\
\text { Skin diseases }\end{array}$ & 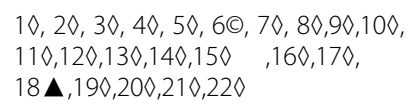 \\
\hline
\end{tabular}


Table 2 Medicinal uses of the reported taxa and their comparison with previous reports (Continued)

\begin{tabular}{|c|c|c|c|c|}
\hline $\begin{array}{l}\text { Scientific name/ } \\
\text { voucher number/ } \\
\text { habit }\end{array}$ & Local name & $\begin{array}{l}\text { Part } \\
\text { used }\end{array}$ & $\begin{array}{l}\text { Method of } \\
\text { preparation/ } \\
\text { mode of } \\
\text { application }\end{array}$ & Diseases treated \\
\hline $\begin{array}{l}\text { Phlomis bracteosa } \\
\text { Royle ex Benth./UZ- } \\
\text { 144/H }\end{array}$ & Cropo & $\begin{array}{l}\text { Lf } \\
\text { Rt }\end{array}$ & $\begin{array}{l}\text { exr, int } \\
\text { pou, int }\end{array}$ & $\begin{array}{l}\text { Fever, Cough } \\
\text { Skin diseases }\end{array}$ \\
\hline $\begin{array}{l}\text { Prunella vulgaris } \mathrm{L} . / \\
\mathrm{UZ}-114 / \mathrm{H}\end{array}$ & Harswa & Wp & exr, int & $\begin{array}{l}\text { Relieve respiratory difficulties, Joint problems, } \\
\text { Gastric spasm }\end{array}$ \\
\hline $\begin{array}{l}\text { Salvia lanata Salisb./ } \\
\text { UZ-127/H }\end{array}$ & Kianar & $\begin{array}{l}\text { Ae } \\
\text { Rt }\end{array}$ & $\begin{array}{l}\text { vef, int } \\
\text { pow, int }\end{array}$ & $\begin{array}{l}\text { Cough, Common cold } \\
\text { Ease bowel evacuation }\end{array}$ \\
\hline $\begin{array}{l}\text { Thymus linearis Benth. } \\
\text { UZ-64/H }\end{array}$ & Chikal & $\begin{array}{l}\text { Wp } \\
\mathrm{Ae}\end{array}$ & $\begin{array}{l}\text { jui, int } \\
\text { pow, int }\end{array}$ & $\begin{array}{l}\text { Stomachache, Liver complaints } \\
\text { Cough }\end{array}$ \\
\hline
\end{tabular}

Previous use reports

$10,20,30,40,50,60,70,80,90,100$, $110,120,130,140,150,160,170,180$ $190,200,210,22 \diamond$

$10,2 \boldsymbol{\Delta}, 3 \boldsymbol{\Delta}, 4 \boldsymbol{\Lambda}, 5 \odot, 60,70,80,90$, $100,110,12 \mathbf{\Lambda}, 130,14 \mathbf{\Lambda}, 150,160,170$, $18 \diamond, 190,20 \diamond, 21 \diamond, 22 \diamond$

$10,2 \boldsymbol{\Lambda}, 3 \boldsymbol{\Lambda}, 4 \boldsymbol{\Lambda}, 50,60,70,80,90$, $100,110,120,130,140,150,160,170$, $180,19 \diamond, 200,210,22 \diamond$

$10,20,3 \boldsymbol{\Lambda}, 4 \boldsymbol{\Lambda}, 50,60,70,80,90$, $10 \mathbf{\Lambda}, 110,12 \mathbf{\Lambda}, 130,14 \mathbf{\Lambda}, 15 \mathbf{\Lambda}, 160$ $170,180,190,200,210,22 \mathbf{\Delta}$

Lythraceae

Punica granatum L./ Darhou

$\begin{array}{ll}\text { Sd } & \text { pow, int } \\ \mathrm{Pl} & \text { jui, int } \\ \mathrm{Fr} & \text { rfo, int }\end{array}$

Stomachache UZ-05/S

$$
\begin{array}{ll}
\text { Fr } & \text { rfo, int } \\
\text { Fr } & \text { exr, int }
\end{array}
$$

Cardiac problems, Dysentery, Diarrhea

$10,20,3 \mathbf{\Delta}, 4 \boldsymbol{\Lambda}, 50,6 \mathbf{\Lambda}, 7 \diamond, 8 \diamond, 90$, $100,110,120,130,140,150,16 \mathbf{\Lambda}, 17 \mathbf{\Lambda}$, $18 \boldsymbol{\Lambda}, 19 \boldsymbol{\Delta}, 20 \diamond, 210,22 \boldsymbol{\Delta}$

Malvaceae

\begin{tabular}{|c|c|c|c|c|}
\hline $\begin{array}{l}\text { Abutilon ramosum } \\
\text { (Cav.) Guill. \& Perr./ } \\
\text { UZ-35/S }\end{array}$ & Shrub & Rt & pow, int & Stomach ailment \\
\hline $\begin{array}{l}\text { Malva parviflora L./ } \\
\text { UZ-45/H }\end{array}$ & Sonchal & Lf & dec, int & Constipation \\
\hline $\begin{array}{l}\text { Malvastrum } \\
\text { coromandelianum (L.) } \\
\text { Garcke/UZ-56/H }\end{array}$ & Bariar & $\mathrm{Ae}$ & dec, int & Kill worms, Dysentery \\
\hline \multicolumn{5}{|l|}{ Meliaceae } \\
\hline $\begin{array}{l}\text { Melia azadirachta L./ } \\
\text { UZ-73/T }\end{array}$ & Daraik & $\begin{array}{l}\mathrm{Fr} / \\
\mathrm{Sd} \\
\mathrm{Lf}\end{array}$ & $\begin{array}{l}\text { pow, int } \\
\text { dec, int }\end{array}$ & $\begin{array}{l}\text { Diabetes, Blood pressure, Blood purifier } \\
\text { Throat infection, Jaundice, Skin problems, Hig } \\
\text { fever }\end{array}$ \\
\hline \multicolumn{5}{|l|}{ Moraceae } \\
\hline $\begin{array}{l}\text { Ficus carica L./UZ- } \\
135 / T\end{array}$ & $\begin{array}{l}\text { Injeer / Barh } \\
\text { Phugwarah }\end{array}$ & $\begin{array}{l}\mathrm{Fr} \\
\mathrm{Lt}\end{array}$ & $\begin{array}{l}\text { rfo, int } \\
\text { pas, ext }\end{array}$ & $\begin{array}{l}\text { Constipation } \\
\text { Wound healing, Extract thrones from skin, } \\
\text { Antidote }\end{array}$ \\
\hline $\begin{array}{l}\text { Ficus palmata Forssk./ } \\
\text { UZ-126/T }\end{array}$ & Phugwarah & $\begin{array}{l}\text { Fr } \\
\text { Lt } \\
\text { Lf } \\
\text { Lf }\end{array}$ & $\begin{array}{l}\text { rfo, int } \\
\text { rub, ext } \\
\text { pas, ext } \\
\text { veg, int }\end{array}$ & $\begin{array}{l}\text { Digestive problems } \\
\text { Extract thorns from skin } \\
\text { Skin problems } \\
\text { Diabetes }\end{array}$ \\
\hline Morus alba L./UZ-96/T & Toot & $\begin{array}{l}\text { Lf } \\
\mathrm{Fr}\end{array}$ & $\begin{array}{l}\text { dec, int } \\
\text { pow, int }\end{array}$ & $\begin{array}{l}\text { Throat inflammation } \\
\text { Cough, Cold, Constipation }\end{array}$ \\
\hline $\begin{array}{l}\text { Morus nigra L./UZ- } \\
116 / T\end{array}$ & Shatoot & $\mathrm{Fr}$ & rfo, int & Cough, Constipation \\
\hline
\end{tabular}

$10,20,30,40,50,60,70,80,90,100$ $110,120,130,140,150,160,170,180$, $190,20 \diamond, 21 \diamond, 22 \diamond$

$10,20,30,40,50,6 \odot, 70,80,90,10 \diamond$, $110,120,130,140,150,160,170,180$, $19 \diamond, 20 \diamond, 21 \odot, 22 \diamond$

$1 \diamond, 20,3 \odot, 4 \odot, 50,6 \boldsymbol{\Delta}, 7 \diamond, 80,90$, $100,110,120,130,140,150,160,170$, $180,190,200,210,220$

$1 \diamond, 2 \diamond, 30,4 \diamond, 50,60,70,80,90,10\rangle$ $11 \mathbf{\Delta}, 120,130,14 \diamond, 150,160,17 \odot, 18 \boldsymbol{\Delta}$ $190,200,21 \mathbf{\Lambda}, 22 \boldsymbol{\Lambda}$

$10,2 \odot, 3 \odot, 4 \odot, 50,60,70,80,90$ $10 \boldsymbol{\Lambda}, 110,120,130,140,150,16 \mathbf{\Lambda}$, $17 \boldsymbol{\Delta}, 180,19 \boldsymbol{\Lambda}, 200,210,22 \boldsymbol{\Delta}$

$10,20,3 \boldsymbol{\Lambda}, 4,5,60,70,80,90,100$ $110,120,13 \odot, 140,150,160,170,18 \odot$, $19 \diamond, 200,21 \diamond, 22 \diamond$

$10,2 \boldsymbol{\Lambda}, 30,40,50,60,70,80,90$ $100,110,120,130,140,150,160,170$, $180,19 \odot, 20 \mathbf{\Lambda}, 21 \mathbf{\Lambda}, 22 \odot$

$10,2 \boldsymbol{\Lambda}, 30,40,50,60,70,80,90$, $100,110,120,130,140,150,160,170$, $18 \mathbf{\Lambda}, 190,200,21 \mathbf{\Lambda}, 22 \odot$

Myrtaceae

Eucalyptus camaldulensis

Safeda Bk rub, ext

Toothache

$1 \diamond, 20,30,40,50,60,70,80,90,10\rangle$, $110,120,130,140,150,160,17 \diamond, 18\rangle$, $190,200,210,220$

Oleaceae

Jasminum officinale Chambili Rt dec, int L./UZ-15/S

Olea ferruginea Wall. Kao Lf dec, int
$1 \diamond, 2 \odot, 3 \diamond, 4 \diamond, 5 \diamond, 6 \boldsymbol{\Lambda}, 7 \diamond, 80,9 \diamond$, 
Table 2 Medicinal uses of the reported taxa and their comparison with previous reports (Continued)

\begin{tabular}{|c|c|c|c|c|c|}
\hline $\begin{array}{l}\text { Scientific name/ } \\
\text { voucher number/ } \\
\text { habit }\end{array}$ & Local name & $\begin{array}{l}\text { Part } \\
\text { used }\end{array}$ & $\begin{array}{l}\text { Method of } \\
\text { preparation/ } \\
\text { mode of } \\
\text { application }\end{array}$ & Diseases treated & Previous use reports \\
\hline ex Aitch./UZ-24/T & & $\begin{array}{l}\mathrm{Ol} \\
\mathrm{Br}\end{array}$ & $\begin{array}{l}\text { exr, int } \\
\text { rfo, ext }\end{array}$ & $\begin{array}{l}\text { Anti-rheumatic } \\
\text { Toothache, Mouth infection }\end{array}$ & $\begin{array}{l}100,110,120,130,140,150,160,17 \odot \\
18 \odot, 19 \diamond, 20 \boldsymbol{\Delta}, 210,22 \boldsymbol{\Delta}\end{array}$ \\
\hline \multicolumn{6}{|l|}{ Onagraceae } \\
\hline $\begin{array}{l}\text { Oenothera rosea L. } \\
\text { Her. ex Aiton/UZ-06/ } \\
\text { H }\end{array}$ & Buti & Lf & inf, int & $\begin{array}{l}\text { Hepatic pain, } \\
\text { Kidney disorders }\end{array}$ & $\begin{array}{l}1 \diamond, 2 \mathbf{\Lambda}, 3 \odot, 4 \odot, 5 \diamond, 6 \mathbf{\Delta}, 7 \diamond, 8 \diamond, 9 \diamond \\
10 \diamond, 11 \diamond, 12 \diamond, 13 \diamond, 14 \diamond, 15 \diamond, 16 \diamond, 17 \diamond \\
18 \diamond, 19 \diamond, 20 \diamond, 21 \diamond, 22 \diamond\end{array}$ \\
\hline \multicolumn{6}{|l|}{ Oxalidaceae } \\
\hline $\begin{array}{l}\text { Oxalis corniculata L./ } \\
\mathrm{UZ}-36 / \mathrm{H}\end{array}$ & Khattiboti & $\begin{array}{l}\text { Wp } \\
\text { Lf } \\
\text { Lf }\end{array}$ & $\begin{array}{l}\text { exr, int } \\
\text { pou, int } \\
\text { dec, int }\end{array}$ & $\begin{array}{l}\text { Diarrhea, Skin diseases, Dysentery, Blood } \\
\text { purification } \\
\text { Inflammation } \\
\text { Cooling property in fever }\end{array}$ & $\begin{array}{l}10,2 \mathbf{\Lambda}, 30,40,5,6 \mathbf{\Lambda}, 70,8 \odot, 9 \mathbf{\Delta} \\
10 \mathbf{\Delta}, 110,12 \mathbf{\Delta}, 130,140,150,160,17 \diamond, \\
18 \mathbf{\Lambda}, 190,20 \diamond, 21 \odot, 22 \mathbf{\Delta}\end{array}$ \\
\hline \multicolumn{6}{|l|}{ Pinaceae } \\
\hline $\begin{array}{l}\text { Pinus roxburghii Sarg./ } \\
\text { UZ-16/T }\end{array}$ & Chir & Rs & pas, ext & $\begin{array}{l}\text { Wound healing, Healing cracks in feet, } \\
\text { Antidote }\end{array}$ & $\begin{array}{l}1,2 \mathbf{\Lambda}, 3 \mathbf{\Lambda}, 4 \mathbf{\Lambda}, 5,6,7,8,90,100 \\
110,120,130,140,150,160,170,18 \mathbf{\Lambda} \\
190,200,210,220\end{array}$ \\
\hline \multicolumn{6}{|l|}{ Plantaginaceae } \\
\hline $\begin{array}{l}\text { Plantago lanceolata } \\
\text { L./UZ-07/H }\end{array}$ & Ispgol & $\begin{array}{l}\text { Lf } \\
\text { Sd }\end{array}$ & $\begin{array}{l}\text { dec, ext } \\
\text { pow, int }\end{array}$ & $\begin{array}{l}\text { Wound inflammation } \\
\text { Throat sores } \\
\text { Constipation }\end{array}$ & $\begin{array}{l}1 \mathbf{\Delta}, 2 \mathbf{\Delta}, 3 \odot, 4 \odot, 50,60,70,80,90 \\
10 \mathbf{\Delta}, 110,12 \mathbf{\Delta}, 130,14 \mathbf{\Delta}, 150,160 \\
170,180,19 \odot, 200,210,22 \mathbf{\Delta}\end{array}$ \\
\hline $\begin{array}{l}\text { Veronica laxa Benth./ } \\
\text { UZ-27/H }\end{array}$ & Sriri & Wp & tea, int & $\begin{array}{l}\text { Nervous system disorder, Respiratory tract, } \\
\text { Cardiovascular system }\end{array}$ & $\begin{array}{l}1 \diamond, 2 \mathbf{\Delta}, 30,40,50,60,70,80,90 \\
10 \diamond, 110,120,130,140,150,160,170 \\
18 \diamond, 190,200,210,22 \diamond\end{array}$ \\
\hline \multicolumn{6}{|l|}{ Poaceae } \\
\hline $\begin{array}{l}\text { Chrysopogon } \\
\text { serrulatus Trin./UZ-86/ } \\
\text { G }\end{array}$ & Bari Gaas & Wp & pas, ext & Used for skin care & 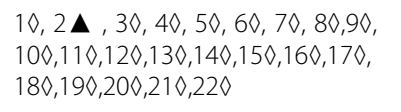 \\
\hline $\begin{array}{l}\text { Cynodon dactylon (L.) } \\
\text { Pers./GUZ-138/G }\end{array}$ & Khabbal & Lf & pas, ext & Muscle and joint fractures & 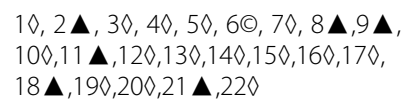 \\
\hline $\begin{array}{l}\text { Dactylis glomerata L./ } \\
\text { UZ-17/G }\end{array}$ & Gadu & $\begin{array}{l}\text { Wp } \\
\text { Wp }\end{array}$ & $\begin{array}{l}\text { pas, int } \\
\text { exr, int }\end{array}$ & $\begin{array}{l}\text { Allergies } \\
\text { Anti-tumor, Kidney ailments, Bladder ailments }\end{array}$ & $\begin{array}{l}1 \diamond, 2 \boldsymbol{\Delta}, 30,40,5 \diamond, 60,7 \diamond, 8 \diamond, 9 \diamond \\
10 \diamond, 11 \diamond, 120,130,140,150,160,17 \diamond \\
180,19 \diamond, 200,21 \diamond, 22 \diamond\end{array}$ \\
\hline $\begin{array}{l}\text { Dichanthium } \\
\text { annulatum (Forssk.) } \\
\text { Stapf/UZ-75/G }\end{array}$ & $\begin{array}{l}\text { Murgah } \\
\text { Ghass }\end{array}$ & Bk & inf, int & Cough & $\begin{array}{l}1 \diamond, 2 \mathbf{\Delta}, 30,40,50,60,70,80,90 \\
10 \diamond, 110,120,130,140,150,160,17 \diamond \\
18 \diamond, 190,200,21 \mathbf{\Delta}, 22 \diamond\end{array}$ \\
\hline $\begin{array}{l}\text { Digitalis ciliata } \\
\text { Trautv./UZ-124/G }\end{array}$ & Diljit & $\mathrm{Sd}$ & pow, int & $\begin{array}{l}\text { Cardiac treatments, Anti-proliferative, Used for } \\
\text { suppressing tumors }\end{array}$ & $\begin{array}{l}10,20,30,40,50,60,70,80,90,100, \\
110,120,130,140,150,160,170,180, \\
190,200,210,22 \diamond\end{array}$ \\
\hline $\begin{array}{l}\text { Echinochloa colona } \\
\text { (L.) Link/UZ-10/G }\end{array}$ & Sanawakri & Wp & inf, int & Hemorrhage problems & $\begin{array}{l}10,20,30,40,50,60,70,80,90,100, \\
110,120,130,140,150,160,170,180, \\
190,200,210,22 \diamond\end{array}$ \\
\hline $\begin{array}{l}\text { Eleusine indica (L.) } \\
\text { Gaertn./UZ-107/G }\end{array}$ & $\begin{array}{l}\text { Madhana } \\
\text { ghass }\end{array}$ & $\begin{array}{l}\text { Rt } \\
\text { Lf } \\
\text { Wp }\end{array}$ & $\begin{array}{l}\text { pou, int } \\
\text { dec, int } \\
\text { exr, int }\end{array}$ & $\begin{array}{l}\text { Gonorrhea } \\
\text { Scanty urination } \\
\text { Fever, Anti-inflammatory, Jaundice }\end{array}$ & $\begin{array}{l}10,20,30,40,50,60,70,80,90,100, \\
110,120,130,140,150,160,170,180, \\
190,200,21 \boldsymbol{\Delta}, 22 \diamond\end{array}$ \\
\hline $\begin{array}{l}\text { Heteropogon } \\
\text { contortus (L.) P. Beauv. } \\
\text { ex Roem. \& Schult./ } \\
\text { UZ-26/G }\end{array}$ & Sariyalaghass & Rt & inf, int & Scanty urination & $\begin{array}{l}1 \diamond, 2 \diamond, 3 \diamond, 4 \diamond, 5 \diamond, 6 \diamond, 7 \diamond, 8 \diamond, 9 \diamond, 10 \diamond, \\
11 \diamond, 12 \diamond, 13 \diamond, 14 \diamond, 15 \diamond, 16 \diamond, 17 \diamond, 18 \diamond, \\
19 \diamond, 20 \diamond, 210,22 \diamond\end{array}$ \\
\hline $\begin{array}{l}\text { Imperata cylindrica (L.) } \\
\text { Raeusch./UZ-115/G }\end{array}$ & Dibb & Wp & exr, int & Asthma, Bruises, Paralysis, Anti-inflammatory & $\begin{array}{l}10,2 \boldsymbol{\Delta}, 30,40,50,60,70,80,90 \\
100,110,120,130,140,15 \diamond, 160,17 \diamond \\
180,190,200,21 \mathbf{\Delta}, 22 \diamond\end{array}$ \\
\hline $\begin{array}{l}\text { Lolium temulentum L./ } \\
\text { UZ-65/G }\end{array}$ & Grass & Sd & pow, int & Sedative & $\begin{array}{l}10,20,30,40,50,60,70,80,90,100, \\
110,120,130,140,150,160,170,180, \\
190,200,21 \diamond, 22 \diamond\end{array}$ \\
\hline
\end{tabular}


Table 2 Medicinal uses of the reported taxa and their comparison with previous reports (Continued)

\begin{tabular}{|c|c|c|c|c|c|}
\hline $\begin{array}{l}\text { Scientific name/ } \\
\text { voucher number/ } \\
\text { habit }\end{array}$ & Local name & $\begin{array}{l}\text { Part } \\
\text { used }\end{array}$ & $\begin{array}{l}\text { Method of } \\
\text { preparation/ } \\
\text { mode of } \\
\text { application }\end{array}$ & Diseases treated & Previous use reports \\
\hline $\begin{array}{l}\text { Setaria pumila (Poir.) } \\
\text { Roem. \& Schult./UZ- } \\
97 / G\end{array}$ & $\begin{array}{l}\text { Kangni, } \\
\text { Loomar Gaas }\end{array}$ & $\begin{array}{l}\text { Lf } \\
\text { Lf } \\
\text { Gr }\end{array}$ & $\begin{array}{l}\text { exr, int } \\
\text { pou, ext } \\
\text { jui, int }\end{array}$ & $\begin{array}{l}\text { Eye drops } \\
\text { Fast healing } \\
\text { Cooling agent }\end{array}$ & $\begin{array}{l}1 \diamond, 2 \diamond, 3 \diamond, 4 \diamond, 5 \diamond, 6 \diamond, 7 \diamond, 8 \diamond, 9 \diamond, 10 \diamond, \\
11 \diamond, 12 \diamond, 130,140,150,16 \diamond, 17 \diamond, 180, \\
19 \diamond, 200,21 \diamond, 22 \diamond\end{array}$ \\
\hline $\begin{array}{l}\text { Themeda anathera } \\
\text { (Nees ex Steud.) } \\
\text { Hack./UZ-98/G }\end{array}$ & Bari ghass & $\mathrm{Ae}$ & pou, ext & Backache, Blood purifier & $\begin{array}{l}1 \diamond, 2 \mathbf{\Delta}, 3 \odot, 4 \odot, 5 \diamond, 6 \diamond, 7 \diamond, 80,9 \diamond \\
10 \diamond, 110,12 \diamond, 13 \diamond, 14 \diamond, 15 \diamond, 16 \diamond, 17 \diamond \\
180,190,20 \diamond, 210,22 \diamond\end{array}$ \\
\hline \multicolumn{6}{|l|}{ Polygalaceae } \\
\hline $\begin{array}{l}\text { Polygala abyssinica R. } \\
\text { Br. ex Fresen./UZ-37/ } \\
\mathrm{H}\end{array}$ & Arna & Rt & jui, ext & Evil eye, Antidote to snake bite & 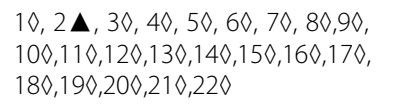 \\
\hline \multicolumn{6}{|l|}{ Polygonaceae } \\
\hline $\begin{array}{l}\text { Persicaria maculosa } \\
\text { Gray/UZ-51/H }\end{array}$ & Ochi & $\begin{array}{l}\text { Sd } \\
\text { Rt } \\
\text { Lf }\end{array}$ & $\begin{array}{l}\text { pow, int } \\
\text { pas, int } \\
\text { pas, ext }\end{array}$ & $\begin{array}{l}\text { Dysentery, Cholera } \\
\text { Scabies } \\
\text { Wound healing }\end{array}$ & $\begin{array}{l}10,20,30,40,50,60,70,80,90,100, \\
110,120,130,140,150,160,170,180, \\
190,200,210,22 \diamond\end{array}$ \\
\hline $\begin{array}{l}\text { Polygonum } \\
\text { ramosissimum Michx./ } \\
\text { UZ-40/H }\end{array}$ & Bannali & Wp & pas, int & Urinary tract infection & $\begin{array}{l}10,20,30,40,50,60,70,80,90,100, \\
110,120,130,140,150,160,170,180, \\
190,200,210,22 \diamond\end{array}$ \\
\hline $\begin{array}{l}\text { Rumex hastatus } \\
\text { D.Don/UZ-59/H }\end{array}$ & Jnglipalak & $\begin{array}{l}\text { Lf } \\
\text { Rt }\end{array}$ & $\begin{array}{l}\text { boi, int } \\
\text { pas, ext }\end{array}$ & $\begin{array}{l}\text { Constipation } \\
\text { Skin disorder }\end{array}$ & 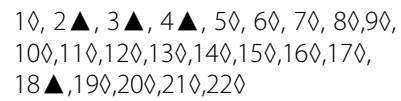 \\
\hline
\end{tabular}

Primulaceae

$\begin{array}{llll}\begin{array}{l}\text { Anagallis arvensis L./ Billibooti } \\ \text { UZ-47/H }\end{array} & \text { Wp exr, int } \\ & & \text { Wp pas, ext } \\ & & \\ \begin{array}{lll}\text { Androsace rotundifolia } \\ \text { Hardw./UZ-58/H }\end{array} & \text { Thandijarri } & \text { Rh } & \text { ext, int } \\ \text { Lf } & \text { inf, int }\end{array}$

Pteridaceae

Adiantum tenerum

Sw./UZ-130/F

Onychium japonicum (Thunb.) Kunze/UZ-

28/F

Pteris vittata L./UZ149/F

\begin{tabular}{|c|c|c|}
\hline Hansraj & $\mathrm{Fd}$ & $\begin{array}{l}\text { jui, int } \\
\text { pas, ext }\end{array}$ \\
\hline Pathba & $\begin{array}{l}\text { Lf \& } \\
\text { Rh }\end{array}$ & jui, int \\
\hline nore & $\mathrm{Fd}$ & pas, ext \\
\hline
\end{tabular}

Wound healing

Ranunculaceae UZ-89/S

Ranunculus arvensis L.NZ-80/H

Ranunculus muricatus L./UZ-91/H

Thalictrum revolutum Beni

DC./UZ-112/H

Rosaceae

Cotoneaster racemiflora Wall. ex Lindl./UZ-08/S
Diabetes

Cough

Asthma

Asthma

Wp jui, int

Blood purifier, Curing fever

\section{Cough, Fever, Dysentery, Ulcers \\ Burning sensation, Epileptic fits \\ Dysentery, Diarrhea problems, Epilepsy \\ Cataract \\ Stomachache, Emetic}

Lowering fever, Depression, Tuberculosis, Liver

Improving the complexion, especially for freckle

$\diamond, 2 \diamond, 3\rangle, 4\rangle, 5\rangle, 6 \diamond, 7\rangle, 8\rangle, 9\rangle, 10\rangle$ $110,120,130,140,150,160,170,18 \diamond$ $190,200,210,220$

$10,2 \boldsymbol{\Lambda}, 3 \odot, 4 \odot, 50,60,70,80,90$, $100,110,12 \mathbf{\Lambda}, 130,140,150,160,170$, $18 \diamond, 19 \diamond, 20 \diamond, 21 \diamond, 22 \diamond$

$1 \diamond, 2 \diamond, 3 \diamond, 4 \diamond, 5 \diamond, 6 \diamond, 7\rangle, 80,90,10\rangle$, $110,120,130,140,150,160,170,180$ $190,200,210,220$

$10,2 \boldsymbol{\Lambda}, 3 \odot, 4 \odot, 50,6 \mathbf{\Delta}, 70,80,90$, $100,110,120,130,140,150,160,170$, $180,190,200,210,220$

$10,20,30,40,50,60,70,80,90,100$, $110,120,130,140,150,160,170,18 \mathbf{\Lambda}$ $190,200,210,220$

$10,20,3 \odot, 4 \odot, 50,6 \odot, 70,80,90$ $100,110,120,130,140,150,160,170$, $180,190,200,210,22$

$10,20,30,40,50,60,70,80,90,10\rangle$, $110,120,130,140,150,160,170,180$

$190,200,210,220$

$10,20,30,40,50,60,70,80,90,100$, $110,120,130,140,150,160,170,180$, $190,200,210,220$ 
Table 2 Medicinal uses of the reported taxa and their comparison with previous reports (Continued)

\begin{tabular}{|c|c|c|c|c|c|}
\hline $\begin{array}{l}\text { Scientific name/ } \\
\text { voucher number/ } \\
\text { habit }\end{array}$ & Local name & $\begin{array}{l}\text { Part } \\
\text { used }\end{array}$ & $\begin{array}{l}\text { Method of } \\
\text { preparation/ } \\
\text { mode of } \\
\text { application }\end{array}$ & Diseases treated & Previous use reports \\
\hline $\begin{array}{l}\text { Duchesnea indica } \\
\text { (Jacks.) Focke/UZ-48/ } \\
\mathrm{H}\end{array}$ & Budimewa & $\begin{array}{l}\mathrm{Fl} \\
\mathrm{Wp}\end{array}$ & $\begin{array}{l}\text { boi, int } \\
\text { exr, int }\end{array}$ & $\begin{array}{l}\text { Blood circulation } \\
\text { Swelling, Boils, Burns }\end{array}$ & $\begin{array}{l}10,2 \mathbf{\Lambda}, 3 \mathbf{\Lambda}, 4 \mathbf{\Lambda}, 50,6 \mathbf{\Lambda}, 70,80 \\
90,100,11 \mathbf{\Lambda}, 120,130,140,150,160 \\
17 \diamond, 180,19 \mathbf{\Lambda}, 200,210,22 \diamond\end{array}$ \\
\hline $\begin{array}{l}\text { Eriobotrya japonica } \\
\text { (Thunb.) Lindl./UZ-29/ } \\
\mathrm{T}\end{array}$ & Loukat & $\begin{array}{l}\text { Wp } \\
\mathrm{Lf} \\
\mathrm{Fr} \\
\mathrm{FI}\end{array}$ & $\begin{array}{l}\text { dec, int } \\
\text { pas, int } \\
\text { rfo, int } \\
\text { boi, int }\end{array}$ & $\begin{array}{l}\text { Cough, Constipation } \\
\text { Nose bleeds, Coughing up blood, Diarrhea, } \\
\text { Depression, Skin diseases, Digestive disorders, } \\
\text { Respiratory problems } \\
\text { Common cold }\end{array}$ & $\begin{array}{l}10,20,3 \mathbf{\Lambda}, 4 \boldsymbol{\Lambda}, 50,60,70,80,90 \\
100,110,120,130,140,150,160,170 \\
180,190,200,210,220\end{array}$ \\
\hline $\begin{array}{l}\text { Fragaria nubicola } \\
\text { (Lindl. ex Hook.f.) } \\
\text { Lacaita/UZ-148/H }\end{array}$ & Boodimava & $\mathrm{Fr}$ & jui, int & Diarrhea, Dysentery, Diabetes, Sexual diseases & $\begin{array}{l}1 \diamond, 2 \mathbf{\Lambda}, 3 \mathbf{\Lambda}, 4 \mathbf{\Delta}, 50,60,7 \mathbf{\Lambda}, 80 \\
90,100,110,120,130,140,150,160,170, \\
180,190,200,210,22 \mathbf{\Delta}\end{array}$ \\
\hline $\begin{array}{l}\text { Potentilla reptans L./ } \\
\text { UZ-137/H }\end{array}$ & Gul bota & Wp & jui, int & Diarrhea, Intestinal infections & $\begin{array}{l}10,20,30,40,50,60,70,80,90,100 \\
110,120,130,140,150,160,170,180 \\
190,200,210,220\end{array}$ \\
\hline $\begin{array}{l}\text { Prunus armeniaca L./ } \\
\text { UZ-70/T }\end{array}$ & $\begin{array}{l}\text { Hari, } \\
\text { Khubani, } \\
\text { Apricot }\end{array}$ & $\begin{array}{l}\mathrm{Fr} \\
\mathrm{Sd}\end{array}$ & $\begin{array}{l}\text { eat, int } \\
\text { oil, ext }\end{array}$ & $\begin{array}{l}\text { Constipation } \\
\text { Softening effect on the skin }\end{array}$ & 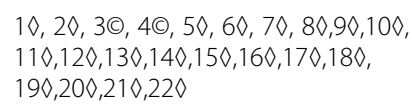 \\
\hline $\begin{array}{l}\text { Prunus domestica L./ } \\
\text { UZ-87/T }\end{array}$ & Alucha & $\mathrm{Fr}$ & eat, int & $\begin{array}{l}\text { Irregular menstruation, Miscarriage, Alcoholic } \\
\text { beverages and liqueur }\end{array}$ & 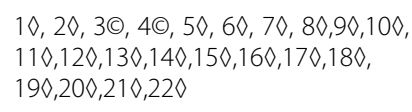 \\
\hline $\begin{array}{l}\text { Prunus persica (L.) } \\
\text { Batsch/UZ-68/T }\end{array}$ & Aruu, Peach & Lf & jui, int & Gastritis, Cough, Bronchitis, Kill worms & $\begin{array}{l}1 \diamond, 2 \diamond, 3 \odot, 4 \odot, 5 \diamond, 6 \mathbf{\Delta}, 7 \diamond, 80,9 \diamond \\
100,110,120,130,140,15 \diamond, 160,17 \diamond \\
18 \mathbf{\Delta}, 190,20 \diamond, 21 \diamond, 22 \diamond\end{array}$ \\
\hline $\begin{array}{l}\text { Pyrus malus L./UZ-99/ } \\
\mathrm{T}\end{array}$ & Saib & $\mathrm{Fr}$ & jui, int & $\begin{array}{l}\text { Rheumatism, Hypertension, Tonic for vigorous } \\
\text { body, Strengthen bones, face spots }\end{array}$ & $\begin{array}{l}1 \diamond, 20,3 \odot, 4 \odot, 5 \diamond, 60,70,80,9 \diamond, 10 \diamond, \\
11 \diamond, 12 \diamond, 13 \diamond, 14 \diamond, 15 \diamond, 160,170,180, \\
19 \diamond, 200,21 \diamond, 22 \diamond\end{array}$ \\
\hline $\begin{array}{l}\text { Pyrus pashia Buch. } \\
\text {-Ham. ex D. Don/UZ- } \\
38 / T\end{array}$ & Tangi & $\begin{array}{l}\mathrm{Fr} \\
\mathrm{Fr}\end{array}$ & $\begin{array}{l}\text { pas, int } \\
\text { eat, int }\end{array}$ & $\begin{array}{l}\text { Dark eye circles } \\
\text { Constipation }\end{array}$ & $\begin{array}{l}1 \diamond, 2 \mathbf{\Delta}, 3 \odot, 4 \diamond, 5 \diamond, 6 \diamond, 7 \diamond, 8 \diamond, 9 \diamond \\
10 \mathbf{\Delta}, 11 \diamond, 12 \diamond, 13 \diamond, 14 \diamond, 15 \diamond, 16 \diamond, 17 \diamond \\
18 \diamond, 19 \diamond, 20 \diamond, 21 \diamond, 22 \diamond\end{array}$ \\
\hline $\begin{array}{l}\text { Rosa brunonii Lindl./ } \\
\text { UZ-121/S }\end{array}$ & Chal & $\begin{array}{l}\mathrm{Fl} \\
\mathrm{FI}\end{array}$ & $\begin{array}{l}\text { pow, int } \\
\text { dec, int }\end{array}$ & $\begin{array}{l}\text { Heart tonic, Skin diseases } \\
\text { Constipation }\end{array}$ & $\begin{array}{l}10,2 \mathbf{\Lambda}, 3 \odot, 4 \odot, 50,6 \odot, 70,80,90 \\
100,110,12 \mathbf{\Lambda}, 130,14 \mathbf{\Lambda}, 150,160,170 \\
18 \odot, 190,200,210,22 \diamond\end{array}$ \\
\hline $\begin{array}{l}\text { Rosa indica L./UZ-30/ } \\
\text { S }\end{array}$ & Galab & $\mathrm{Fl}$ & exr, int & Eye diseases, Stomachache, Fever, Pneumonia & $\begin{array}{l}1 \diamond, 2 \diamond, 30,40,50,60,70,80,90,100 \\
11 \diamond, 120,130,140,150,160,170,18 \mathbf{\Delta} \\
190,200,210,220\end{array}$ \\
\hline $\begin{array}{l}\text { Rubus ellipticus Sm./ } \\
\text { UZ-109/S }\end{array}$ & Aakhara & $\begin{array}{l}\text { Lf } \\
\text { Rt / } \\
\text { Bk }\end{array}$ & $\begin{array}{l}\text { dec, int } \\
\text { pow, int } \\
\text { exr, int }\end{array}$ & $\begin{array}{l}\text { Diarrhea, Bleeding } \\
\text { Against skin diseases especially female genitalia } \\
\text { Dysentery }\end{array}$ & 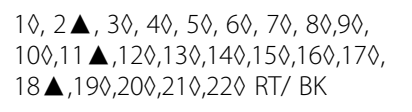 \\
\hline $\begin{array}{l}\text { Rubus niveus Thunb./ } \\
\text { UZ-19/S }\end{array}$ & Garachi & $\begin{array}{l}\text { Lf } \\
\text { Rt }\end{array}$ & $\begin{array}{l}\text { pow, int } \\
\text { dec, int }\end{array}$ & $\begin{array}{l}\text { Diarrhea, Fever, Blood purifier } \\
\text { Dysentery, Colic, Pain, Whooping Cough }\end{array}$ & 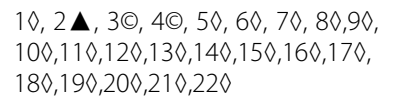 \\
\hline $\begin{array}{l}\text { Spiraea canescens D. } \\
\text { Don/UZ-57/H }\end{array}$ & Jhar, Mariala & $\begin{array}{l}\text { Rt } \\
\text { Sd }\end{array}$ & $\begin{array}{l}\text { exr, int } \\
\text { pow, int }\end{array}$ & $\begin{array}{l}\text { Enema and to treat venereal conditions } \\
\text { Insomnia }\end{array}$ & $\begin{array}{l}10,2 \mathbf{\Lambda}, 30,40,50,60,70,80,90 \\
100,11 \diamond, 120,130,140,150,160,170 \\
180,190,200,210,220\end{array}$ \\
\hline
\end{tabular}

Galium aparine L./UZ- Boora Wp mix, int $119 / \mathrm{H}$

Rutaeae

Zanthoxylum armatum DC./UZ$106 / S$

Timber

Wp jui, int

$\begin{array}{ll}\text { Sd / } & \text { rfo, int } \\ \text { Bk } & \text { rub, int } \\ \text { Bk } & \end{array}$

Tonic, Cholera, fever, Dyspepsia, Stomachache Toothache

Constipation, Stomachic diseases Scanty urination, Constipation

Arthritis, Gout, Lower back pain, Urinary complaints, Gout, Digestive disorders, Liver
$10,2 \mathbf{\Lambda}, 3 \mathbf{\Lambda}, 4 \mathbf{\Lambda}, 50,60,7 \mathbf{\Lambda}, 8,90$, $10 \mathbf{\Lambda}, 110,120,130,140,150,160,170$ $180,190,200,210,220$

$10,2 \mathbf{\Lambda}, 3 \odot, 4 \odot, 50,6 \mathbf{\Lambda}, 70,80,90$ $10 \boldsymbol{\Lambda}, 11 \boldsymbol{\Lambda}, 120,130,140,150,160,170$, $18 \odot, 19 \diamond, 20 \diamond, 210,22 \diamond$

$10,2 \boldsymbol{\Lambda}, 30,40,50,60,70,80,90$, $100,110,120,130,140,150,160,17\rangle$. 
Table 2 Medicinal uses of the reported taxa and their comparison with previous reports (Continued)

\begin{tabular}{|c|c|c|c|c|c|}
\hline $\begin{array}{l}\text { Scientific name/ } \\
\text { voucher number/ } \\
\text { habit }\end{array}$ & Local name & $\begin{array}{l}\text { Part } \\
\text { used }\end{array}$ & $\begin{array}{l}\text { Method of } \\
\text { preparation/ } \\
\text { mode of } \\
\text { application }\end{array}$ & Diseases treated & Previous use reports \\
\hline & & Lf & pas, ext & $\begin{array}{l}\text { disorders, Fever, Relieve the pain of menstrual } \\
\text { cramps } \\
\text { Hemorrhoids } \\
\text { Infected wounds and sprains, Caries of teeth } \\
\text { and bones }\end{array}$ & $18 \diamond, 19 \diamond, 20 \diamond, 21 \diamond, 22 \diamond$ \\
\hline
\end{tabular}

Sapindaceae

$\begin{array}{lllll}\begin{array}{l}\text { Aesculus indica (Wall. } \\ \text { ex Camb.) Hook./UZ- } \\ 141 / T\end{array} & \text { Bankhore } & \mathrm{Fr} & \text { rfo, int } & \begin{array}{l}\text { Colic, Rheumatism } \\ \text { Leucorrhoea }\end{array} \\ \begin{array}{l}\text { Dodonaea viscosa (L.) } \\ \text { Snathaa }\end{array} & \text { Lf } & \text { pow, int } & \text { pow, ext } & \text { Healing agent }\end{array}$
Jacq./UZ-139/S

Scrophulariaceae

Verbascum thapsus L./ Gidar UZ-18/H

Simaroubaceae

Ailanthus altissima

(Mill.) Swingle/UZ-92/

Draviyae

$\mathrm{T}$

Solanaceae

Solanum villosum

Mill./UZ-09/H

Kaach, Lf dec, int

Maach $\quad$ Fr rfo, Int

Urticaceae

Debregeasia salicifolia

(D. Don) Rendle/UZ-

39/S

Urtica dioica L./UZ-49/ Kinjii

$\mathrm{H}$

Valerianaceae

Valeriana jatamansi Mushk bala Rh exr, int Jones./UZ-60/H

Verbenaceae

Vitex agnus-castus L./

$\mathrm{UZ}-88 / \mathrm{T}$

Verbena officinalis L./ Chandni Wp inf, int $\mathrm{UZ}-67 / \mathrm{H}$

Violaceae

Viola odorata L./UZ- Banafshan WP - Woi, int 100/H

Eczema, Dermatitis $\begin{array}{ll}\text { Lf } & \text { dec, ext } \\ \text { Wp inf, int }\end{array}$

Rt ext, int

Bk inf, int

Lf ext, int

Ae inf, ext

Rt inf, int

Rt pas, int

Scanty urination

Anti-allergic

Sunburn, Ulcers, Tumors, Piles, Sedative,

Narcotic

Toothache, Relieve cramps, Convulsions

Dysentery, Diarrhea

Blood purifier

Scanty urination

Tongue infection in children

Intestinal pain, Neurosis, Constipation

Female reproductive system disorders, Scanty urination, Digestive disorders, Anxiety,

Stomachache

Scanty urination, Reduces inflammation,

Control bleeding, Malaria, Nervous exhaustion, Depression, Asthma, Migraine, Jaundice
$1 \diamond, 2 \diamond, 3 \odot, 4 \odot, 50,60,70,80,90$,

$10 \boldsymbol{\Delta}, 110,120,130,140,150,160,170$, $18 \diamond, 190,20 \odot, 21 \diamond, 22 \diamond$

$10,20,30,40,50,60,70,80,90,100$, $110,120,130,140,150,160,17 \diamond, 18 \odot$

$190,200,210,220$

$10,2 \mathbf{\Lambda}, 3 \mathbf{\Lambda}, 4 \boldsymbol{\Lambda}, 50,6 \mathbf{\Lambda}, 70,80$ $90,10 \boldsymbol{\Lambda}, 110,120,13 \mathbf{\Lambda}, 140,150,160$, $170,18 \boldsymbol{\Delta}, 19 \diamond, 20 \diamond, 21 \diamond, 22 \diamond$

$10,2 \boldsymbol{\Lambda}, 30,40,50,60,70,80,90$, $100,110,120,130,140,150,160,170$ $180,190,200,210,22 \Delta$

$1 \diamond, 2 \diamond, 3 \diamond, 4 \diamond, 5 \diamond, 6 \diamond, 7 \diamond, 8 \diamond, 9 \diamond, 10 \diamond$, $110,120,130,140,150,160,170,180$ $190,200,21 \diamond, 22 \diamond$

$10,20,3 \odot, 4 \odot, 50,6 \odot, 70,80,90$, $100,110,120,130,140,150,160,170$ $18 \odot, 19 \diamond, 200,210,220$

$10,20,30,40,50,60,7 \mathbf{\Lambda}, 80,90$

$100,11 \mathbf{\Lambda}, 120,13 \mathbf{\Lambda}, 140,15 \mathbf{\Lambda}, 160$

$170,180,190,200,210,22 \mathbf{\Delta}$

$10,20,3 \boldsymbol{\Lambda}, 4 \boldsymbol{\Lambda}, 50,60,7 \boldsymbol{\Lambda}, 80,90$, $10 \boldsymbol{\Lambda}, 110,12 \mathbf{\Lambda}, 130,140,150,160,170$, $180,19 \diamond, 200,210,22 \mathbf{\Delta}$

$1 \diamond, 2 \diamond, 3 \diamond, 4 \diamond, 5 \diamond, 6 \diamond, 7 \diamond, 8 \diamond, 9 \diamond, 10 \diamond$, $110,120,130,140,150,160,17$

Stomachache $0,180,190,200,210$, 220

$10,2 \boldsymbol{\Lambda}, 30,40,50,60,70,80,90$, $100,110,120,130,140,150,160,170$, $18 \diamond, 19 \diamond, 200,21 \diamond, 22 \diamond$

$10,20,30,40,5 \mathbf{\Lambda}, 60,70,80,90$ $10 \boldsymbol{\Lambda}, 110,120,130,14 \diamond, 150,16 \mathbf{\Lambda}, 17\rangle$, $180,190,200,210,220$

Habit: H, Herbs; S, Shrubs; T, Trees; F, Ferns; E, Epiphyte. Part(s) Used: Lf, Leaf; Fr, Fruit; Rt, Root; St, Stem; Ae, Aerial Parts; Wp, Whole Plant; Fd, Fronds; Sd, Seed; Fl, Flower; Bk, Bark; Bl, Bulb; Rh, Rhizome; In, Inflorescence; Sh, Shoot; Lt, Latex; Br, Branches; Rs, Resin; Gr, Grain; Pd, Pods; Pl, Pulp; Ol, Oil. Method of preparation: pow, Powder; dec, Decoction; ext, Extract; pas, Paste; jui, Juice; pou, Poultice; inf, Infusion; che, Chewed; veg, Vegetable; rub, Rubbing; eat, Eaten; coo, Cooked; boi, Boiled; flu, Fluid; fra, Fragrance, mix, Mixture. Mode of Aministration: int, Internal; exr, External. Previous use reports: $(\Theta)=$ plants with similar use(s); $(\mathbf{\Lambda})=$ plants with dissimilar use (s); ( ()$=$ plants not reported in previous study; bold written highlights the frequent use for a given plant. 1: Mahmood et al. [64]; 2: Khan et al. [65]; 3: Amjadet al [41];; 4: Shaheenet al [47].; 5: Ishtiaq et al. [66]; 6: Amjad et al. [67]; 7: Gilani et al. [68]; 8: Gulshan et al. [69]; 9: Mahmood et al. [70];10: Rana et al. [71]; 11: Jadhav [72];12: Gidey et al. [73]; 13: Dar [74];14: Bano et al. [39]; 15: Khan et al. [75]; 16: Hussain et al. [76] 17: Ullah and Bibi [77]; 18: Qaseem et al. [40]; 19: Aziz et al. [14]; 20. Ahmad et al. [32]; Umair et al. [78]; 22. Hussain et al. [76] 


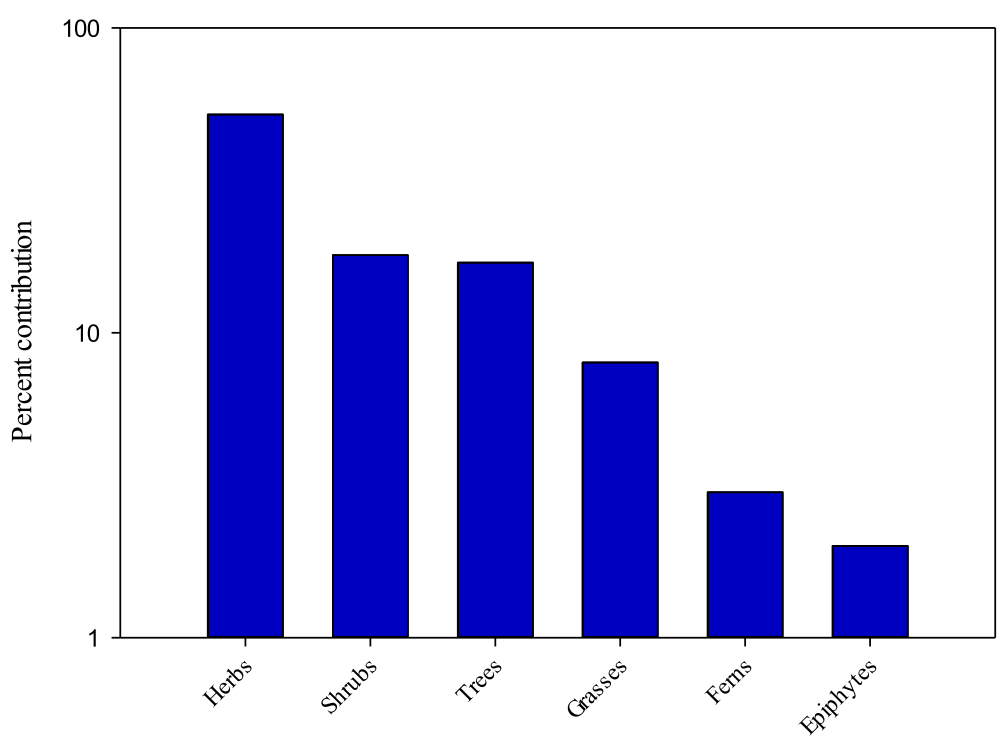

Life form

Fig. 3 Life form distribution pattern of the reported plant species in the study area

plants used in the treatment of common illnesses. According to Heinrich [103], high ICF can help in identifying potentially effective medicinal plants. It was observed that in our study, the highest agreement level was recorded for diseases reported as the most widespread in rural communities of the Bagh district and other areas of Pakistan. The digestive disorder was also reported as first use class by other ethnic communities across world [102, 104-109]. The prevalence of digestive disorders among the local inhabitant might be due to inadequate

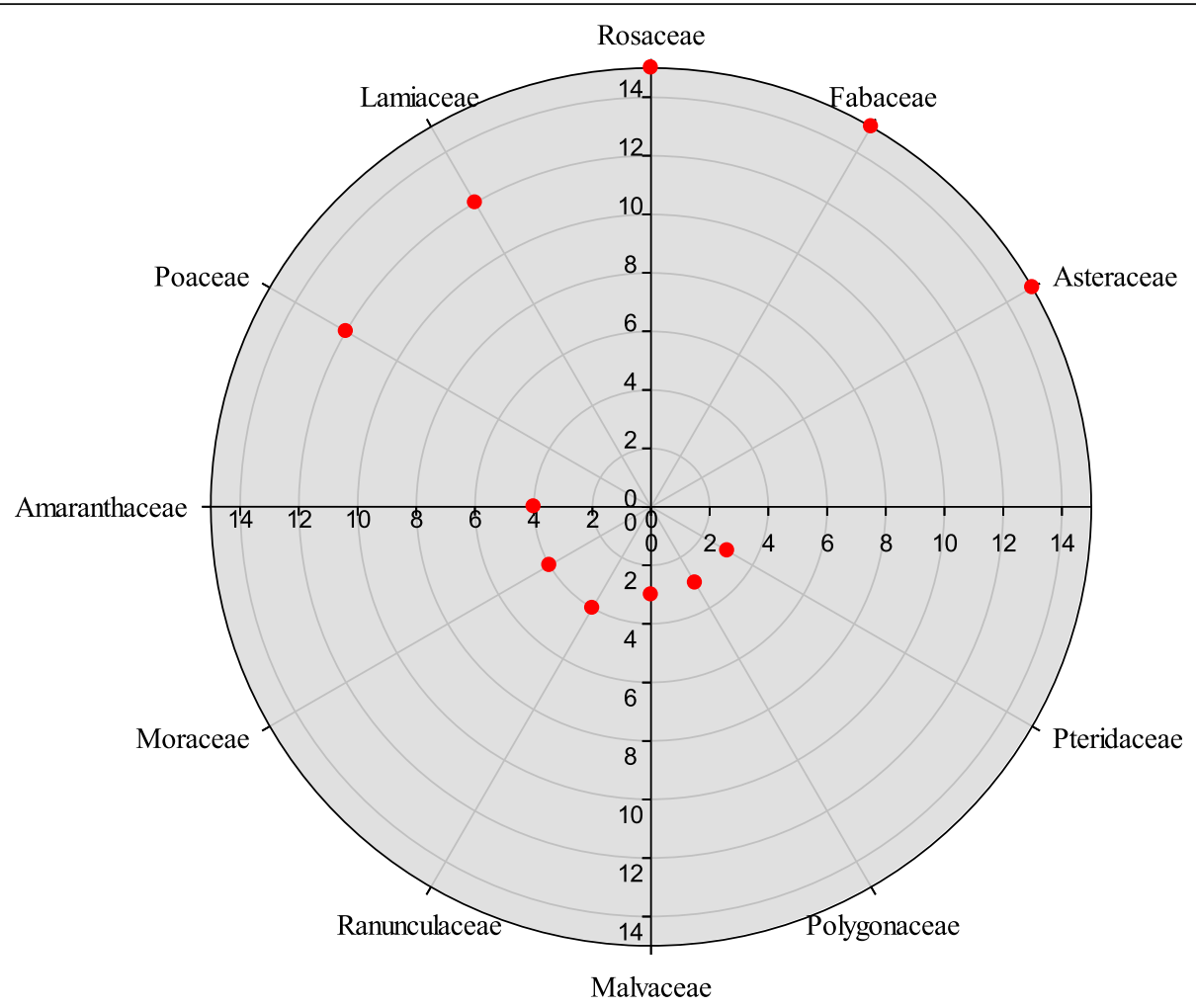

Fig. 4 Family contribution of medicinal flora in the study area 


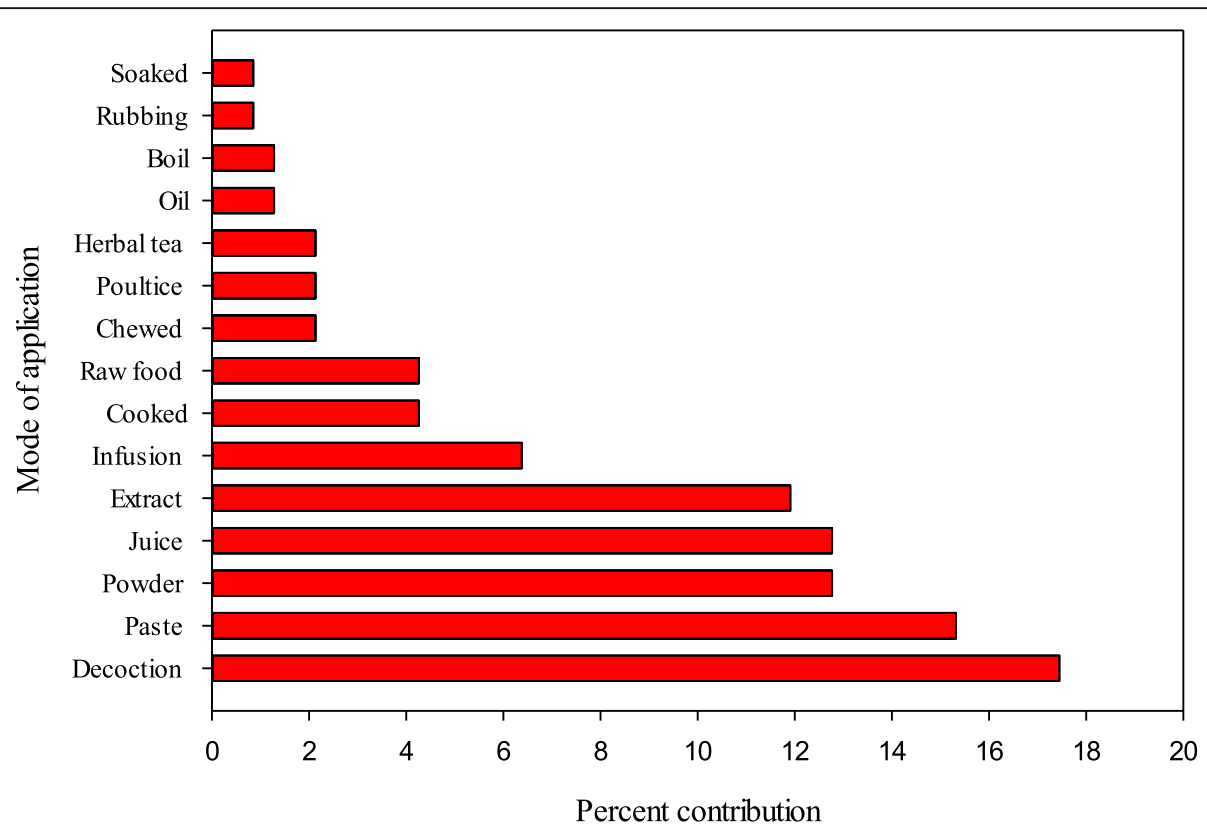

Fig. 5 Method of preparation of herbal recipes

availability of hygienic food and drinking water and also the common inhalation of fuel wood's smoke [40, 42, 110, 111]. Moreover, the devastating earthquake of 2005 caused extensive damage to water resources (freshwater springs) and water supply schemes, causing the drinking water quality to be very poor, with local communities usually using contaminated water. The second highest ICF was recorded for respiratory disorders which may be due to prevalence of cold and moist conditions at high altitude Kayani et al. [20]. Besides, study area is a rich

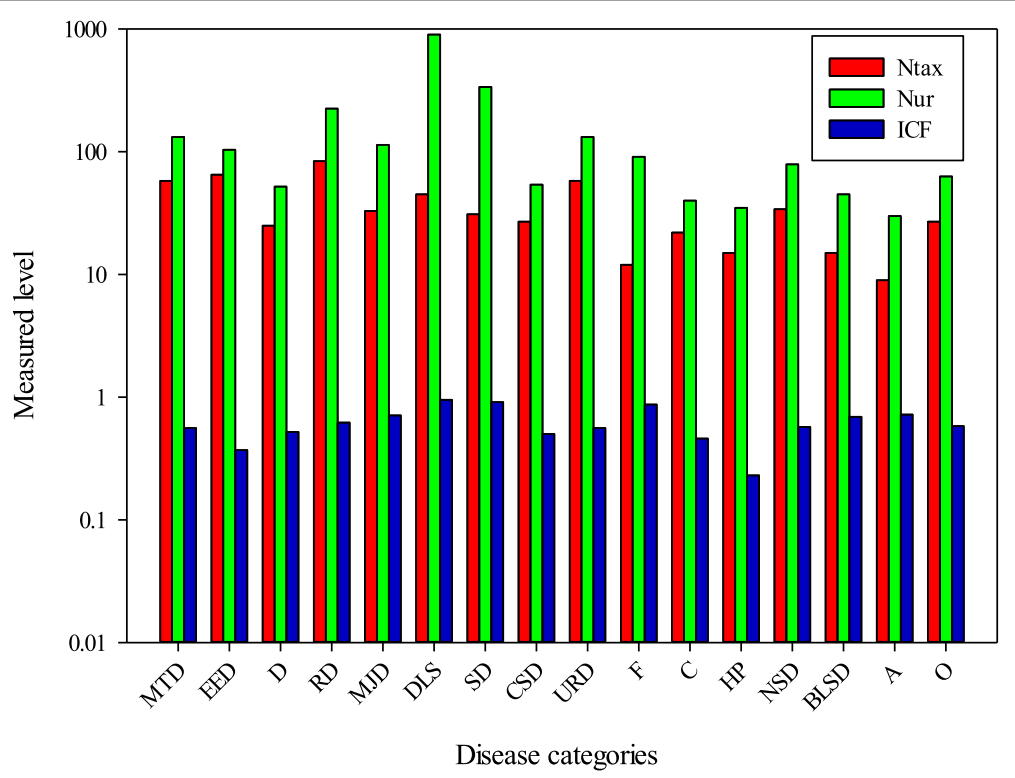

Fig. 6 Informant consensus factor of diseases with use reports and total number of species used. Ntax total species used by all the informants for group of ailments, UR total number of use reports in each group of disease, ICF informant consensus factor, MTD mouth-throat diseases, EED eye and ear diseases, D diabetes, RD respiratory diseases, MJD muscular and joint diseases, DLS digestive system and liver diseases, SD skin diseases, CSD circulatory system diseases, URD urinary and reproductive system diseases, F fever, C cancer, HP hair problem, NSD nervous system disorder, $B L S D$ blood and lymphatic system diseases, $A$ antidotes, $O$ others 
source of flowering plants and mushrooms and the prevalence of pollen and spore present in air also cause respiratory problem. Skin disorders also have high ICF value, and UV radiations, unhygienic conditions, and combine family systems (where many members live together in one room or home even some time with domesticated animals) could be the possible reasons of the prevalence of skin infections in the study area.

\section{Relative frequency of citation and use value}

Relative frequency of citation (RFC) is used to identify the highly important species in various ailments as cited by local people [31]. The value of RFC ranged between 0.93 and 0.04. Berberis lycium had highest RFC value (0.81). Other plant species with significant RFC value were Ajuga bracteosa, Prunella vulgaris, Adiantum capillus-veneris, Desmodium polycarpum, Pinus roxburgii, Rosa brunonii, Punica granatum, Zanthoxylum armatum, and Jasminum mesnyi (Table 3). The plants species with high RFC value were abundant in the area therefore the local people were much familiar with them particularly with reference to ethnomedicinal perspective over a long time period. Likewise, the plants with special properties to cure particular disease were well known among the local culture; therefore, their precise properties to treat particular disease have got famous and deep rooted. The plant species with high RFC values would be interesting for phytochemical and pharmacological profiling and possible future drug discovery, as well as authentication at a commercial level $[20,40,44,112]$.

Use value reflects the relative importance of every species with reference to more use reports cited by local informants. The use value ranged between 1.05 and 0.08 . Mentha longifolia (1.05), Olea ferouginea (1.02), and Zanthoxylum armatum (1.01) had high use value while other species with significantly high use value were Solanum villosum (0.93), Cynoglossom lanceolatum (0.94), Rosa indica (0.95), and Punica granatum (0.97) (Table 3). UV value is directly related with use reports. Plant species with more use reports have high use value and vice versa $[40,41,95]$. These plant species are used in repetitive manner and are biologically more active [113]. It is not necessary that the plant which has low UV value become unimportant or not biologically active as the RFC and UV are constant in particular area but they may be change according to the variation in the knowledge of indigenous people from area to area or within area.

Species with high RFC and UV show high healing potential for particular disease. Species with high RFC and UV were often overharvested by inhabitants, so they are prioritized for conservation and sustainable use; otherwise, they will be extinct from the area in near future $[20,44,114]$. The ethnomedicinal knowledge is at risk because there might be no resource left for younger generations. The main reason for this is that the local inhabitants of the area, especially young generations, have little interest and understanding or knowledge about ethnomedicinal plants, and are already dependent upon allopathic medicine for their healthcare $[47,114,115]$.

\section{Relative importance}

Relative importance value is used to determine the diversity of a species for treating various ailments. Galium aparine (96) and Mimosa pudica (91) had highest RI values while Verbena officinalis (81.5), Fragaria nubicola (81), Verbascum thapsus (79), and Melia azadirachta (73) had high RI values (Table 3). It was observed that species with high RI value was used frequently for treating several ailments. The natives have too much ethnomedicinal knowledge regarding these plants. Therefore, importance of these species increase as the number of treated systems increases $[41,114,116]$.

\section{Fidelity level}

The fidelity value reflects the preference of particular plant species as reported by local people for curing particular ailment in the area. The FL value of reported species ranged from 18.2 to $100 \%$. Fidelity values of four plant species viz Mentha longifolia, Punica granatum, Zanthoxylum armatum, and Olea ferruginea were found $100 \%$, and these species were used to cure stomachache, dysentery, rheumatism, and other digestive disorders. Other medicinal plants having high FL value were Solanum villosum (93.8), Cynoglossum lanceolatum (91.8), Dalbergia sissoo (83.8), Bidens biternata (85.7\%), Rubus ellipticus (86.5\%), and Melia azadirachta (84.6\%) (Fig. 7 ). These species were mostly used to cure the digestive problems like diarrhea, dysentery, indigestion, stomachache and gastrointestinal pain, etc. High FL of a species reflects extensive use of a specific plant species to treat a specific disease dominant in area [13, 17, 114]. Species with high FL value are important model plants which can be subjected to further pharmacological studies [20, $44,118]$. Some other studies in literature also recoded high fidelity level for species used to cure digestive problems $[13,114,119]$. The species with low FL values were not well known by the natives in term of ethnomedicinal knowledge. This forecast that may be in upcoming generation the ethnobotanical knowledge about these plant species may be completely depleted $[44,114,120]$ (Table 4).

\section{Novel uses}

In this study, we compared our results with 22 published papers from adjoining and areas with similar vegetation across Pakistan and world. The highest values for the Jaccard Index (JI) were result of the studies published by Amjad et al. [41] and Shaheen et al. [47] on Toil peer (AJK) and Perl valley respectively. The least value for JI 
Table 3 Quantitative analysis of ethnobotanical data

\begin{tabular}{|c|c|c|c|c|c|c|c|}
\hline Scientific name & FC & RFC & Ui & UV & RelPH & RelBS & RI \\
\hline Abutilon ramosum & 3 & 0.04 & 3 & 0.04 & 0.08 & 0.13 & 10.5 \\
\hline Acacia modesta & 30 & 0.4 & 33 & 0.44 & 0.17 & 0.25 & 21 \\
\hline Acacia nilotica & 64 & 0.86 & 65 & 0.87 & 0.42 & 0.38 & 40 \\
\hline Achillea millefolium & 38 & 0.15 & 46 & 0.62 & 0.5 & 0.75 & 62.5 \\
\hline Achyranthes aspera & 50 & 0.67 & 66 & 0.88 & 0.25 & 0.38 & 31.5 \\
\hline Adiantum tenerum & 19 & 0.25 & 21 & 0.28 & 0.58 & 0.63 & 60.5 \\
\hline Aesculus indica & 34 & 0.45 & 39 & 0.52 & 0.33 & 0.5 & 41.5 \\
\hline Ajuga bracteosa & 42 & 0.56 & 54 & 0.72 & 0.42 & 0.5 & 46 \\
\hline Ailanthus altissima & 60 & 0.81 & 64 & 0.86 & 0.25 & 0.25 & 25 \\
\hline Allium griffithianum & 42 & 0.56 & 48 & 0.64 & 0.25 & 0.13 & 19 \\
\hline Alternanthera pungens & 10 & 0.13 & 23 & 0.31 & 0.17 & 0.13 & 15 \\
\hline Amaranthus spinosus & 36 & 0.48 & 39 & 0.52 & 0.25 & 0.25 & 25 \\
\hline Amaranthus viridis & 51 & 0.68 & 57 & 0.77 & 0.42 & 0.5 & 46 \\
\hline Anagallis arvensis & 22 & 0.29 & 29 & 0.39 & 0.5 & 0.5 & 50 \\
\hline Anaphalis adnata & 14 & 0.18 & 18 & 0.24 & 0.17 & 0.25 & 21 \\
\hline Androsace rotundifolia & 6 & 0.08 & 11 & 0.14 & 0.25 & 0.38 & 31.5 \\
\hline Angelica glauca & 20 & 0.27 & 27 & 0.36 & 0.17 & 0.25 & 21 \\
\hline Artemisia vulgaris & 52 & 0.7 & 55 & 0.74 & 0.17 & 0.25 & 12.67 \\
\hline Asplenium dalhousiae & 34 & 0.45 & 34 & 0.45 & 0.08 & 0.13 & 10.5 \\
\hline Astragalus Canadensis & 8 & 0.1 & 8 & 0.1 & 0.08 & 0.13 & 0.5 \\
\hline Bauhinia variegate & 44 & 0.59 & 47 & 0.63 & 0.25 & 0.25 & 50 \\
\hline Berberis lycium & 60 & 0.81 & 67 & 0.9 & 0.25 & 0.38 & 31.5 \\
\hline Bidens biternata & 53 & 0.71 & 61 & 0.82 & 0.17 & 0.25 & 12.65 \\
\hline Callicarpa mycrophylla & 39 & 0.52 & 43 & 0.58 & 0.42 & 0.5 & 46 \\
\hline Cannabis sativa & 58 & 0.78 & 60 & 0.81 & 0.33 & 0.5 & 41.5 \\
\hline Capsella bursa-pastoris & 25 & 0.33 & 44 & 0.59 & 0.33 & 0.38 & 35.5 \\
\hline Carissa opaca & 32 & 0.43 & 38 & 0.51 & 0.33 & 0.38 & 35.5 \\
\hline Cirsium vulgare & 32 & 0.43 & 41 & 0.55 & 0.25 & 0.38 & 31.5 \\
\hline Chenopodium album & 19 & 0.25 & 19 & 0.25 & 0.08 & 0.13 & 10.5 \\
\hline Chrysopogon serrulatus & 4 & 0.05 & 4 & 0.05 & 0.08 & 0.13 & 10.5 \\
\hline Clematis grata & 2 & 0.027 & 2 & 0.027 & 0.17 & 0.25 & 21 \\
\hline Commelina benghalensis & 5 & 0.06 & 5 & 0.08 & 0.13 & & 10.5 \\
\hline Convolvulus arvensis & 52 & 0.7 & 58 & 0.78 & 0.58 & 0.63 & 60.5 \\
\hline Conyza Canadensis & 40 & 0.54 & 47 & 0.63 & 0.58 & 0.5 & 54 \\
\hline Cornus macrophylla & 8 & 0.1 & 12 & 0.16 & 0.08 & 0.13 & 10.5 \\
\hline Cotoneaster racemiflora & 2 & 0.02 & 2 & 0.02 & 0.17 & 0.25 & 21 \\
\hline Crepis multicaulis & 10 & 0.13 & 10 & 0.13 & 0.08 & 0.13 & 10.5 \\
\hline Crotalaria juncea & 2 & 0.02 & 4 & 0.05 & 0.33 & 0.5 & 41.5 \\
\hline Cuscuta reflexa & 57 & 0.77 & 60 & 0.81 & 0.17 & 0.25 & 21 \\
\hline Cynodon dactylon & 60 & 0.81 & 64 & 0.86 & 0.08 & 0.13 & 10.5 \\
\hline Cynoglossum lanceolatum & 69 & 0.93 & 70 & 0.94 & 0.08 & 0.13 & 10.5 \\
\hline Cyperus rotundus & 4 & 0.05 & 9 & 0.12 & 0.25 & 0.13 & 19 \\
\hline Dactylis glomerata & 6 & 0.08 & 19 & 0.25 & 0.42 & 0.63 & 52.5 \\
\hline Dalbergia sissoo & 60 & 0.81 & 62 & 0.83 & 0.5 & 0.63 & 56.5 \\
\hline
\end{tabular}


Table 3 Quantitative analysis of ethnobotanical data (Continued)

\begin{tabular}{|c|c|c|c|c|c|c|c|}
\hline Scientific name & FC & RFC & $\mathrm{Ui}$ & UV & RelPH & RelBS & RI \\
\hline Debregeasia salicifolia & 33 & 0.44 & 38 & 0.51 & 0.17 & 0.25 & 21 \\
\hline Desmodium elegans & 26 & 0.35 & 26 & 0.35 & 0.17 & 0.25 & 21 \\
\hline Dichanthium annulalum & 10 & 0.13 & 10 & 0.13 & 0.17 & 0.25 & 21 \\
\hline Dicliptera bupleuroides & 10 & 0.13 & 20 & 0.27 & 0.25 & 0.38 & 31.5 \\
\hline Digitalis ciliata & 8 & 0.1 & 16 & 0.21 & 0.25 & 0.38 & 31.5 \\
\hline Dodonaea viscosa & 60 & 0.81 & 60 & 0.81 & 0.25 & 0.38 & 31 \\
\hline Dryopteris filix-mas & 60 & 0.81 & 60 & 0.81 & 0.08 & 0.13 & 10.5 \\
\hline Duchesnea indica & 30 & 0.4 & 38 & 0.51 & 0.17 & 0.25 & 21 \\
\hline Echinochloa colona & 2 & 0.02 & 4 & 0.05 & 0.17 & 0.25 & 21 \\
\hline Elaeagnus umbellate & 42 & 0.56 & 54 & 0.72 & 0.33 & 0.38 & 35.5 \\
\hline Eleusine indica & 9 & 0.12 & 14 & 0.18 & 0.42 & 0.63 & 52.5 \\
\hline Eriobotrya japonica & 61 & 0.82 & 63 & 0.85 & 0.33 & 0.5 & 41.5 \\
\hline Eucalyptus camaldulensis & 43 & 0.58 & 46 & 0.62 & 0.08 & 0.13 & 6.33 \\
\hline Euphorbia helioscopia & 64 & 0.86 & 68 & 0.91 & 0.25 & 0.25 & 25 \\
\hline Ficus carica & 61 & 0.82 & 63 & 0.85 & 0.33 & 0.38 & 19.08 \\
\hline Ficus palmate & 50 & 0.67 & 70 & 0.94 & 0.42 & 0.5 & 25.42 \\
\hline Fragaria nubicola & 46 & 0.62 & 48 & 0.64 & 1.00 & 0.63 & 81.5 \\
\hline Galium aparine & 54 & 0.72 & 56 & 0.75 & 0.92 & 1.00 & 96.0 \\
\hline Geranium rotundifolium & 46 & 0.62 & 49 & 0.66 & 0.25 & 0.38 & 31.5 \\
\hline Gerbera gossypina & 38 & 0.51 & 40 & 0.54 & 0.42 & 0.38 & 40.0 \\
\hline Hedera nepalensis & 20 & 0.27 & 35 & 0.47 & 0.25 & 0.38 & 31.5 \\
\hline Helianthus annuus & 50 & 0.67 & 61 & 0.82 & 0.58 & 0.63 & 60.5 \\
\hline Heteropogon contortus & 10 & 0.13 & 14 & 0.18 & 0.17 & 0.25 & 21.0 \\
\hline Impatiens edgeworthii & 14 & 0.18 & 25 & 0.33 & 0.25 & 0.38 & 31.5 \\
\hline Imperata cyilindrica & 7 & 0.09 & 24 & 0.32 & 0.42 & 0.5 & 46 \\
\hline Indigofera linifolia & 24 & 0.32 & 28 & 0.37 & 0.08 & 0.13 & 10.5 \\
\hline Ipomoea purpurea & 49 & 0.66 & 52 & 0.7 & 0.33 & 0.5 & 41.5 \\
\hline Jasminum officinale & 54 & 0.72 & 59 & 0.79 & 0.33 & 0.5 & 25.33 \\
\hline Juglans regia & 58 & 0.78 & 66 & 0.89 & 0.17 & 0.25 & 21 \\
\hline Justicia adhatoda & 40 & 0.54 & 53 & 0.71 & 0.33 & 0.25 & 29 \\
\hline Lathyrus aphaca & 15 & 0.2 & 17 & 0.22 & 0.08 & 0.13 & 0.5 \\
\hline Launaea procumbens & 15 & 0.2 & 27 & 0.36 & 0.17 & 0.25 & 21 \\
\hline Lespedeza juncea & 5 & 0.06 & 8 & 0.1 & 0.17 & 0.13 & 15 \\
\hline Lolium temulentum & 2 & 0.02 & 3 & 0.04 & 0.08 & 0.13 & 10.5 \\
\hline Lotus corniculatus & 5 & 0.06 & 7 & 0.09 & 0.5 & 0.75 & 62.7 \\
\hline Malva parviflora & 65 & 0.87 & 65 & 0.87 & 0.08 & 0.13 & 0.5 \\
\hline Malvastrum coromandelianum & 42 & 0.56 & 48 & 0.64 & 0.17 & 0.13 & 5 \\
\hline Maytenus nemorosa & 4 & 0.05 & 7 & 0.09 & 0.17 & 0.25 & 21 \\
\hline Medicago polymorpha & 44 & 0.59 & 49 & 0.66 & 0.17 & 0.13 & 15 \\
\hline Melia azadrachta & 65 & 0.87 & 69 & 0.93 & 0.58 & 0.88 & 73 \\
\hline Mentha spicata & 55 & 0.74 & 57 & 0.77 & 0.17 & 0.25 & 21 \\
\hline Mentha longifolia & 64 & 0.86 & 78 & 1.05 & 0.5 & 0.38 & 44 \\
\hline Micromeria biflora & 33 & 0.44 & 53 & 0.71 & 0.17 & 0.13 & 15 \\
\hline Mimosa pudica & 3 & 0.04 & 5 & 0.06 & 0.83 & 0.1 & 91.5 \\
\hline
\end{tabular}


Table 3 Quantitative analysis of ethnobotanical data (Continued)

\begin{tabular}{|c|c|c|c|c|c|c|c|}
\hline Scientific name & FC & RFC & $\mathrm{Ui}$ & UV & RelPH & RelBS & RI \\
\hline Morus alba & 61 & 0.82 & 66 & 0.89 & 0.33 & 0.38 & 35.5 \\
\hline Morus nigra & 44 & 0.59 & 60 & 0.81 & 0.25 & 0.25 & 50 \\
\hline Nerium oleander & 50 & 0.67 & 54 & 0.72 & 0.5 & 0.63 & 56.5 \\
\hline Ocimum sanctum & 55 & 0.74 & 57 & 0.77 & 0.17 & 0.25 & 21 \\
\hline Oenothera rosea & 15 & 0.2 & 20 & 0.27 & 0.17 & 0.25 & 35.5 \\
\hline Olea ferruginea. & 64 & 0.86 & 76 & 1.02 & 0.58 & 0.75 & 38.08 \\
\hline Onychium japonicum & 17 & 0.22 & 24 & 0.32 & 0.17 & 0.13 & 15 \\
\hline Origanum vulgare & 16 & 0.21 & 28 & 0.37 & 0.25 & 0.25 & 50 \\
\hline Otostegia limbata & 3 & 0.04 & 8 & 0.1 & 0.17 & 0.13 & 15 \\
\hline Oxalis corniculata & 60 & 0.81 & 65 & 0.87 & 0.5 & 0.5 & 50 \\
\hline Persicaria maculosa & 7 & 0.09 & 15 & 0.2 & 0.42 & 0.38 & 40 \\
\hline Phlomis bracteosa & 8 & 0.1 & 11 & 0.14 & 0.25 & 0.25 & 50 \\
\hline Pinus roxburghii & 60 & 0.81 & 64 & 0.86 & 0.25 & 0.25 & 50 \\
\hline Plantago lanceolata & 30 & 0.4 & 36 & 0.48 & 0.25 & 0.25 & 50 \\
\hline Polygala abyssinica & 2 & 0.02 & 2 & 0.02 & 0.08 & 0.13 & 10.5 \\
\hline Polygonatum geminiflorum & 6 & 0.08 & 11 & 0.16 & 0.58 & 0.5 & 54 \\
\hline Polygonum ramosissimum & 5 & 0.06 & 5 & 0.06 & 0.08 & 0.13 & 10.5 \\
\hline Populus nigra & 53 & 0.71 & 56 & 0.75 & 0.08 & 0.13 & 10.5 \\
\hline Potentilla reptans & 4 & 0.05 & 6 & 0.08 & 0.17 & 0.13 & 15 \\
\hline Prunella vulgaris & 31 & 0.41 & 48 & 0.64 & 0.25 & 0.38 & 31.5 \\
\hline Prunus armeniaca & 35 & 0.47 & 38 & 0.51 & 0.25 & 0.38 & 31.5 \\
\hline Prunus domestica & 39 & 0.52 & 42 & 0.56 & 0.33 & 0.25 & 29 \\
\hline Prunus persica & 43 & 0.58 & 49 & 0.66 & 0.42 & 0.5 & 46 \\
\hline Pteris vittata & 21 & 0.28 & 27 & 0.36 & 0.08 & 0.13 & 10.5 \\
\hline Punica granatum & 68 & 0.91 & 72 & 0.97 & 0.42 & 0.38 & 40 \\
\hline Pyrus malus & 46 & 0.62 & 51 & 0.68 & 0.5 & 0.5 & 50 \\
\hline Pyrus pashia & 38 & 0.51 & 38 & 0.51 & 0.33 & 0.25 & 29 \\
\hline Ranunculus arvensis & 29 & 0.39 & 29 & 0.39 & 0.08 & 0.13 & 10.5 \\
\hline Rauanculus muricatus & 20 & 0.27 & 20 & 0.27 & 0.08 & 0.13 & 1050 \\
\hline Ricinus communis & 28 & 0.37 & 51 & 0.68 & 0.5 & 0.5 & 50 \\
\hline Rosa brunoni & 50 & 0.67 & 52 & 0.7 & 0.25 & 0.38 & 31.5 \\
\hline Rosa indica & 69 & 0.93 & 71 & 0.95 & 0.08 & 0.13 & 0.5 \\
\hline Rubus ellipticus & 58 & 0.78 & 60 & 0.81 & 0.42 & 0.5 & 46 \\
\hline Rubus niveus & 55 & 0.74 & 59 & 0.79 & 0.67 & 0.63 & 65 \\
\hline Rumex hastatus & 51 & 0.68 & 55 & 0.74 & 0.17 & 0.25 & 21 \\
\hline Salvia lanata & 18 & 0.24 & 28 & 0.37 & 0.25 & 0.25 & 50 \\
\hline Sarcococca saligna & 2 & 0.02 & 6 & 0.08 & 0.25 & 0.38 & 31.5 \\
\hline Setaria pumila & 6 & 0.08 & 13 & 0.17 & 0.25 & 0.38 & 31.5 \\
\hline Silybum marianum & 25 & 0.33 & 53 & 0.71 & 0.67 & 0.63 & 65 \\
\hline Solanum villosum & 66 & 0.89 & 69 & 0.93 & 0.17 & 0.25 & 21 \\
\hline Sonchus oleraceus & 52 & 0.7 & 52 & 0.7 & 0.08 & 0.13 & 10.5 \\
\hline Spiraea canescens & 14 & 0.18 & 17 & 0.22 & 0.17 & 0.25 & 21 \\
\hline Swertia cordata & 3 & 0.04 & 9 & 0.12 & 0.33 & 0.25 & 29 \\
\hline Tagetes minuta & 14 & 0.2 & 25 & 0.35 & 0.09 & 0.41 & 115 \\
\hline
\end{tabular}


Table 3 Quantitative analysis of ethnobotanical data (Continued)

\begin{tabular}{|c|c|c|c|c|c|c|c|}
\hline Scientific name & FC & RFC & $\mathrm{Ui}$ & UV & RelPH & RelBS & RI \\
\hline Taraxacum officinale & 54 & 0.72 & 59 & 0.79 & 0.25 & 0.38 & 31.5 \\
\hline Thalictrum revolutum & 11 & 0.14 & 16 & 0.21 & 0.17 & 0.25 & 21 \\
\hline Themeda anathera & 16 & 0.21 & 18 & 0.24 & 0.17 & 0.25 & 21 \\
\hline Thymus linearis & 22 & 0.29 & 39 & 0.52 & 0.25 & 0.25 & 50 \\
\hline Torilis japonica & 14 & 0.18 & 19 & 0.25 & 0.5 & 0.63 & 32 \\
\hline Trichodesma indicum & 14 & 0.18 & 19 & 0.24 & 0.25 & 0.38 & 31.5 \\
\hline Trifolium resupinatum & 20 & 0.27 & 27 & 0.36 & 0.08 & 0.13 & 10.5 \\
\hline Urtica dioica & 44 & 0.59 & 47 & 0.63 & 0.17 & 0.25 & 21 \\
\hline Valeraina jatamansi & 26 & 0.35 & 30 & 0.4 & 0.25 & 0.25 & 25 \\
\hline Verbascum Thapsus & 10 & 0.13 & 12 & 0.16 & 0.83 & 0.75 & 79 \\
\hline Verbena officinalis & 25 & 0.33 & 27 & 0.36 & 0.75 & 0.88 & 81 \\
\hline Veronica laxa & 9 & 0.12 & 13 & 0.17 & 0.83 & 0.75 & 79 \\
\hline Viburnum grandiflorum & 53 & 0.71 & 57 & 0.77 & 0.17 & 0.25 & 21 \\
\hline Vicia sativa & 44 & 0.59 & 51 & 0.68 & 0.83 & 0.88 & 85.5 \\
\hline Vincetoxicum hirundinaria & 15 & 0.2 & 28 & 0.37 & 0.17 & 0.13 & 15 \\
\hline Viola odorata & 62 & 0.83 & 65 & 0.87 & 0.25 & 0.25 & 25 \\
\hline Vitex agnus-castus & 54 & 0.72 & 58 & 0.78 & 0.50 & 0.5 & 50 \\
\hline Xanthium strumarium & 62 & 0.83 & 128 & 1.72 & 0.33 & 0.38 & 35.5 \\
\hline Zanthoxylum armatum & 68 & 0.91 & 75 & 1.01 & 0.42 & 0.5 & 46 \\
\hline
\end{tabular}

FC frequency of citation, RFC relative frequency of citation, Ui use reports cited by each respondent for given species, UV use value, Rel. $P H$ relative number of pharmacological properties attributed to a single plant, Rel. BS relative number of body systems treated by a single species, $R I$ relative importance

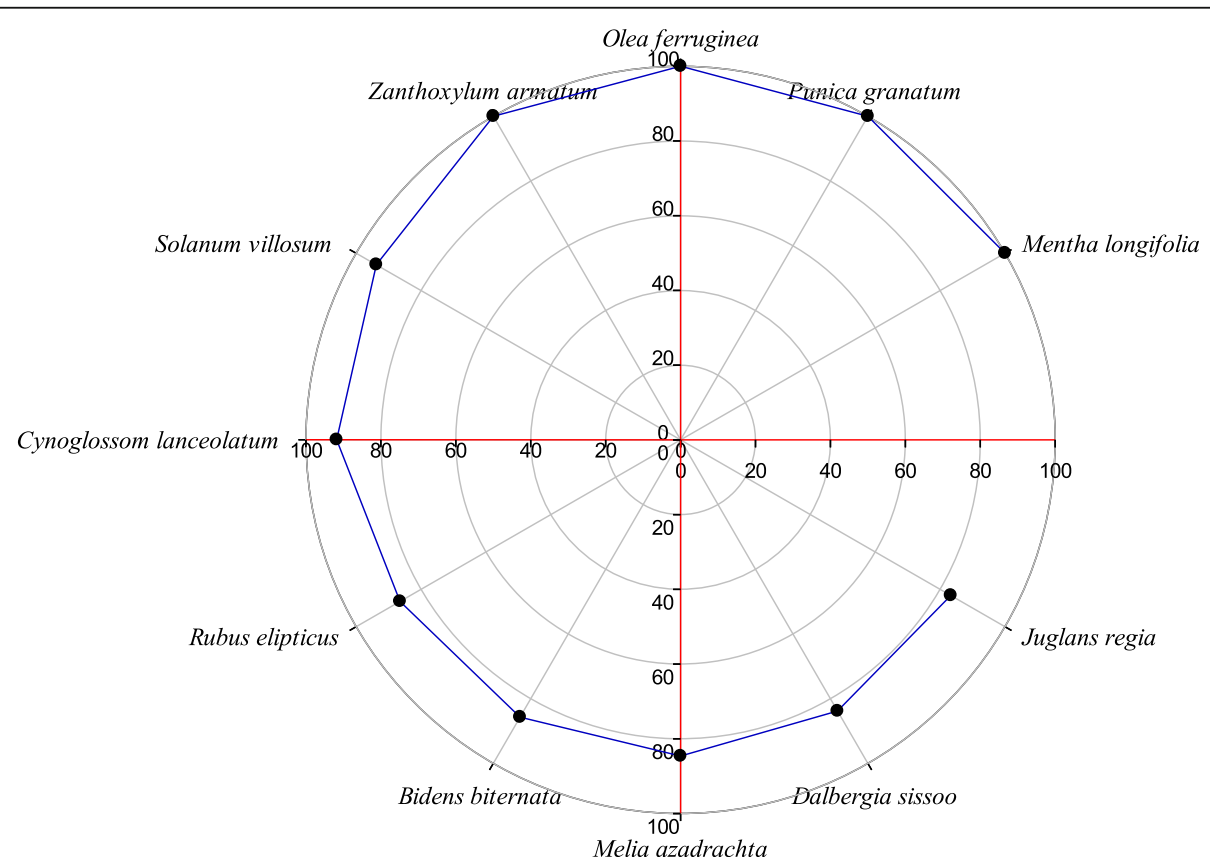

Fig. 7 Top ranked plant species with above $80 \%$ fidelity 
Table 4 Jaccard index comparing the present study with previous articles

\begin{tabular}{|c|c|c|c|c|c|c|c|c|c|c|c|}
\hline Area & SY & NRPs & NPSU & NPDU & TSCBA & SEAA & SESA & PPSU & PPDU & ال & $\mathrm{C}$ \\
\hline \multicolumn{12}{|l|}{ From Azad Jammu \& Kashmir } \\
\hline Toil peer (AJK), Pakistan & 2017 & 121 & 27 & 18 & 45 & 76 & 105 & 22.3 & 14.8 & 33.08 & {$[41]$} \\
\hline Perl valley (AJK), Pakistan & 2017 & 136 & 29 & 21 & 50 & 86 & 100 & 21.3 & 15.4 & 36.7 & {$[47]$} \\
\hline Neelum Valley (AJK), Pakistan & 2011 & 40 & 2 & 7 & 9 & 31 & 141 & 5 & 17.5 & 5.2 & [64] \\
\hline Kotli, AJK, Pakistan & 2017 & 202 & 21 & 19 & 40 & 162 & 110 & 10.3 & 9.4 & 17.2 & [67] \\
\hline Bhimber (AJK), Pakistan & 2011 & 38 & 3 & 8 & 11 & 27 & 139 & 7.8 & 21.05 & 7.09 & [70] \\
\hline Khahuta (AJK), Pakistan & 2013 & 45 & 5 & 9 & 14 & 31 & 136 & 11.1 & 20 & 9.15 & {$[66]$} \\
\hline Muzaffarabad (AJK), Pakistan & 2014 & 52 & 5 & 13 & 18 & 34 & 132 & 9.61 & 25 & 12.1 & [74] \\
\hline Kotli (AJK), Pakistan & 2019 & 80 & 11 & 24 & 35 & 45 & 105 & 13.7 & 30 & 30.4 & {$[40]$} \\
\hline Sharda, Neelum Valley (AJK), Pakistan & 2012 & 39 & 2 & 9 & 11 & 28 & 139 & 5.1 & 23 & 7.05 & {$[32]$} \\
\hline \multicolumn{12}{|l|}{ From KPK } \\
\hline Kabal valley (KPK), Pakistan & 2015 & 45 & 2 & 10 & 12 & 33 & 138 & 4.4 & 22.2 & 7.54 & {$[65]$} \\
\hline Skardu valley, Karakoram-Himalayan range, Pakistan & 2014 & 50 & 1 & 4 & 5 & 45 & 145 & 2 & 8 & 2.7 & [39] \\
\hline Ayubia National Park, Abbottabad, Pakistan & 2006 & 21 & 3 & 4 & 7 & 14 & 143 & 14.2 & 19.04 & 4.66 & [68] \\
\hline Tormik valley, Baltistan, Pakistan & 2015 & 63 & 0 & 3 & 3 & 60 & 147 & 0 & 4.76 & 1.47 & [75] \\
\hline Northern Pakistani Afghan borders & 2018 & 92 & 2 & 18 & 20 & 72 & 148 & 2.17 & 19.5 & 10 & [76] \\
\hline Malakand KPK, Pakistan & 2018 & 25 & 2 & 7 & 9 & 16 & 141 & 8 & 28 & 6.08 & [77] \\
\hline Mohmand Agency (FATA), Pakistan & 2018 & 64 & 1 & 14 & 15 & 49 & 135 & 1.56 & 21.8 & 8.87 & [14] \\
\hline \multicolumn{12}{|l|}{ From other areas of Pakistan } \\
\hline Wazirabad, Punjab, Pakistan & 2018 & 31 & 0 & 4 & 4 & 27 & 146 & 0 & 12.9 & 2.36 & [117] \\
\hline Chenab, Punjab, Pakistan & 2019 & 129 & 3 & 24 & 27 & 102 & 123 & 2.32 & 18.6 & 13.6 & [78] \\
\hline Dera Ghazi Khan, Punjab, Pakistan & 2012 & 66 & 7 & 9 & 16 & 50 & 134 & 10.6 & 13.6 & 9.52 & {$[69]$} \\
\hline \multicolumn{12}{|l|}{ From rest of world } \\
\hline Sikles area, Nepal & 2015 & 42 & 2 & 5 & 7 & 35 & 143 & 4.76 & 11.9 & 4.09 & [71] \\
\hline Sangli, Maharashtra, India & 2015 & 21 & 0 & 3 & 3 & 18 & 147 & 0 & 14.2 & 1.85 & {$[72]$} \\
\hline Kunama ethnic group in Northern Ethiopia & 2015 & 115 & 1 & 3 & 4 & 111 & 146 & 0.86 & 2.6 & 1.58 & [73] al., 2015 \\
\hline
\end{tabular}

SY study year, number of reported plant species; NPSU number of plants with similar uses; NPDU number of plants with different uses; TSCBA total species common in both area; SEAA Species enlisted in aligned areas; SESA species enlisted only in study area; PPSU percentage of plant with similar uses; PPDU percentage of plant with different uses; $J$ Jaccard index; $C$ citation

was found in the studies of Jadhava et al. [72] on Sangli, Maharashtra, India, and Gidey et al. [73] on the Kunama ethnic group in Northern Ethiopia. High similarity reflects similar culture, traditions vegetation, and geography among the areas along with high level of cross-cultural exchange of traditional knowledge among the community while high differences or least value of JI reflects that areas do not share common cultural values. Further, the ethnoecological knowledge is often specifically influenced by origin and culture of indigenous communities.

The comparative study of current findings with reported research revealed some novel uses which were not reported earlier from this region. These included the use of the extract of the whole plant of Crepis multicaulis and Maytenus nemorosa to treat eye infections. An extract of the aerial parts of Swertia cordata was used to treat hepatic disorders. Leaves of Cotoneaster racemiflora were used to stop bleeding and pus. The root extract of Spiraea canescens is was to as enema to treat venereal conditions. A bark infusion of Dichanthium annulatum was used to cure cough. A pasted based on the whole plant of Polygonum ramosissimum was used to treat urinary tract infections. The seeds of Persicaria maculosa were used in powdered form to treat cholera.

\section{Threats to medicinal plants and indigenous knowledge}

The majority of the local inhabitant in the rural areas of Harighal are illiterate and their main source of income are agriculture and livestock. Some of them collect medicinal plants and sell them at very low prices to local herb sellers. The herbal sellers export herbs to pharmaceutical companies. Over-exploitation of medicinal plant species by untrained collectors, e.g., uprooting of medicinal plants, forest fires, deforestation, over-grazing, and urbanization, are contributing significantly toward the decline of medicinal plant species of the study area, and 
may finally lead to their extinction. Therefore, authorities should take strict control over protection, conservation, and sustainable utilization of economic plants of the study area. Furthermore, universities, agriculture extension department, and local management may contribute significantly to promote the cultivation of medicinal plants in the area; this will definitely improve the socioeconomic condition of local people of the area.

The traditional practices are highly affected by exposure to modern pharmaceuticals and changing lifestyles. The traditional knowledge about medicinal plants in the study area is gradually declining because this knowledge is now mainly restricted to the older members of the community members which are passing away. The younger generation is not interested in learning about traditional plant use, and makes more use of allopathic medicine. The traditional health practitioners (Hakeems) have profound traditional knowledge, but many are not willing to share it with other people. These factors may lead to the erosion of traditional medicinal knowledge among the rural communities of area.

\section{Conclusion}

This study is the first to report the traditional uses of indigenous medicinal plants from the remote areas of tehsil Harighal, Bagh. The documented data reflect that local people are still highly dependent on medicinal plants for treating various diseases, as public health facilities are hard to reach, and still have a large knowledge of medicinal plants. The traditional knowledge is mainly in the hand of elder people and health practitioners (hakims), but the young generation is not much interested in herbal recipes. This lack of interest, as well as impacts like overgrazing, deforestation, and soil erosion, are reducing the medicinal flora in the area, and strategies related to resource conservation and further ethnobotanical and pharmacological research are highly recommended for the conservation of this precious treasure.

\section{Supplementary information}

Supplementary information accompanies this paper at https://doi.org/10. 1186/s13002-020-00417-w.

Additional file 1:. Appendix l: Cultivation status and endemism of medicinal flora of Tehsil Harighal.

Additional file 2:. Appendix II: Emic and etic use reports of medicinal flora of Harighal.

\section{Acknowledgements}

The authors are thankful to the local inhabitants of the area for sharing knowledge. Taxonomical assistance provided by Dr. M. Ilyas is also duly acknowledged.

\section{Authors' contributions}

MSA, UZ, and AMA designed project, involve in data collection, analysis, and prepared final draft of manuscript. MA and SMHG helped in statistical analysis. RBU completely revised and commented the manuscript. All authors read and approved the final manuscript.

\section{Funding}

No funding was provided by any source to conduct this survey

Availability of data and materials

All data are included in the manuscript

\section{Competing interest}

The authors declare that they have no competing interest.

\section{Ethics approval and consent to participate}

The present research is purely based on field surveys. The ethical approval to conduct the study was given by the Ethics Committee of the Women University of Azad Jammu \& Kashmir, Bagh before initiating the surveys. In addition to this, legal permission to conduct interviews was given by members of municipality committee. A Prior Informed Consent form (PIC) was signed by all the informants after explaining the objective and consequence of study. The PIC was translated into local Pahari language. The ethical guidelines provided by the International Society of Ethnobiology (http://www.ethnobiology.net/) were strictly followed.

\section{Consent for publication}

Not applicable

\section{Author details}

${ }^{1}$ Department of Botany, Women University of Azad Jammu \& Kashmir, Bagh 12500, Pakistan. ${ }^{2}$ Department of Ethnobotany, Institute of Botany, Ilia State University, Tbilisi, Georgia. ${ }^{3}$ Department of Zoology, Women University of Azad Jammu \& Kashmir, Bagh 12500, Pakistan. ${ }^{4}$ Department of

Environmental Sciences, COMSATS University Islamabad, Abbottabad Campus, Abbottabad 22060, Pakistan.

Received: 19 May 2020 Accepted: 6 October 2020

Published online: 27 October 2020

\section{References}

1. Arshad M, Ahmad M, Ahmed E, Saboor A, Abbas A, Sadiq S. An ethnobiological study in Kala Chitta hills of Pothwar region, Pakistan: multinomial logit specification. J Ethnobiol Ethnomed. 2014;10:13.

2. Ford RI. The nature and status of ethnobotany. Michigan: Museum of Anthropology, University of Michigan; 1978.

3. Verpoorte R, Choi YH, Kim HK. Ethnopharmacology and systems biology: a perfect holistic match. J Ethnopharmacol. 2005;100(1-2):53-6.

4. Silva FS, Ramos MA, Hanazaki N, UPd A. Dynamics of traditional knowledge of medicinal plants in a rural community in the Brazilian semi-arid region. Revista Brasileira de Farmacognosia. 2011;21(3):382-91.

5. Cox PA. Will tribal knowledge survive the millennium? Science. 2000; 287(5450):44-5.

6. Khan MPZ, Ahmad M, Zafar M, Sultana S, Ali MI, Sun H. Ethnomedicinal uses of edible wild fruits (EWFs) in Swat Valley, Northern Pakistan. J Ethnopharmacol. 2015;173:191-203.

7. Shabir A, Naveed I, Uneeza J, Noor U, Hina J, Farhat Y. Ethno botanical Wisdom of Inhabitant of Devi Galli Azad Kashmir. Biomedcal J Sci Technol Res. 2017;1(6):1618-27.

8. Shinwari ZK. Medicinal plants research in Pakistan. J Med Plants Res. 2010; 4(3):161-76.

9. Nasir S, Ahmed J, Asrar M. Medicinal plants: a promising resource for poverty alleviation in the milieu of Swat. FUUAST J Biol. 2014;4(2):237-45.

10. Khatun MA, Harun-Or-Rashid M, Rahmatullah M. Scientific validation of eight medicinal plants used in traditional medicinal systems of Malaysia: a review. American-Eurasian J Sustainable Agriculture. 2011;5(1):67-75

11. Shi Q, Li L, Huo C, Zhang M, Wang Y. Study on natural medicinal chemistry and new drug development. Zhongcaoyao - Chinese Traditional Herbal Drugs. 2010;41(10):1583-9.

12. Anna L. Plants for people. London: Oxford University press; 1990.

13. Srithi K, Balslev H, Wangpakapattanawong P, Srisanga P, Trisonthi C. Medicinal plant knowledge and its erosion among the Mien (Yao) in northern Thailand. J Ethnopharmacol. 2009;123(2):335-42. 
14. Aziz MA, Adnan M, Khan AH, Shahat AA, Al-Said MS, Ullah R. Traditional uses of medicinal plants practiced by the indigenous communities at Mohmand Agency, FATA, Pakistan. J Ethnobiol Ethnomed. 2018;14(1):2.

15. Heinrich M, Kufer J, Leonti M, Pardo-de-Santayana M. Ethnobotany and ethnopharmacology —Interdisciplinary links with the historical sciences. J Ethnopharmacol. 2006;107(2):157-60.

16. Kassaye KD, Amberbir A, Getachew B, Mussema Y. A historical overview of traditional medicine practices and policy in Ethiopia. Ethiopian J Health Development. 2006;20(2):127-34.

17. Bibi T, Ahmad M, Tareen RB, Tareen NM, Jabeen R, Rehman SU, Zafar M. Ethnobotany medicinal Plants in district Mastung of Balochistan provincePakistan. J Ethnopharmacol. 2014;157(157):79-89.

18. Veeresham C. Natural products derived from plants as a source of drugs. J Advanc Pharmaceutical Technol Res. 2012;3:200.

19. Mahdi JG. Medicinal potential of willow: A chemical perspective of aspirin discovery. J Saudi Chemical Soc. 2010;14:317-22.

20. Kayani S, Ahmad M, Zafar M, Sultana S, Khan MPZ, Ashraf MA, Hussain J, Yaseen $\mathrm{G}$. Ethnobotanical uses of medicinal plants for respiratory disorders among the inhabitants of Gallies-Abbottabad, Northern Pakistan. J Ethnopharmacol. 2014;156:47-60.

21. Ahmad A, Ali A, Basit A. Ethnomedicinal study of various plants in lone valley, district Chitral, KPK, Pakistan. J Med Plants. 2019;7(3):24-8.

22. Yaseen G, Ahmad M, Shinwari S, Potter D, Zafar M, Zhang G, Shinwari ZK, Sultana S. Medicinal plants diversity used for livelihood of public health in desert and arid regions of Sindh, Pakistan. Pakistan J Botany. 2019;2(31):2409-19.

23. Pieroni A. Local plant resources in the ethnobotany of Theth, a village in the Northern Albanian Alps. Genetic Resources and Crop Evolution. 2008;55: 1197-214.

24. Ankli A, Sticher O, Heinrich M. Medical ethnobotany of the Yucatec Maya: healers' consensus as a quantitative criterion. Econ Botany. 1999:53:144-60.

25. Heinrich M, Gibbons S. Ethnopharmacology in drug discovery: an analysis of its role and potential contribution. J Pharmacy Pharmacol. 2001;53(4):425-32.

26. Malik AY, Singh D. Ethnobotanical and ethnoveterinary importance of plants of scrub areas of Dachigam national park, Jammu and Kashmir, India. Asian J Pharmaceutical Clin Res. 2019;12(3):582-6.

27. Vačkář D, ten Brink B, Loh J, Baillie JE, Reyers B. Review of multispecies indices for monitoring human impacts on biodiversity. Ecological Indicators. 2012;17:58-67.

28. Quave $\mathrm{CL}$, Pieroni A. A reservoir of ethnobotanical knowledge informs resilient food security and health strategies in the Balkans. Nature Plants 2015;1(2):14021

29. Adnan M, Ullah I, Tariq A, Murad W, Azizullah A, Khan AL, Ali N. Ethnomedicine use in the war affected region of northwest Pakistan. J Ethnobiol Ethnomedicine. 2014;10(1):16.

30. Amiri MS, Joharchi MR. Ethnobotanical investigation of traditional medicinal plants commercialized in the markets of Mashhad, Iran. Avicenna J Phytomed. 2013;3(3):254

31. Vitalini S, Iriti M, Puricelli C, Ciuchi D, Segale A, Fico G. Traditional knowledge on medicinal and food plants used in Val San Giacomo (Sondrio, Italy) —an alpine ethnobotanical study. J Ethnopharmacol. 2013; 145(2):517-29.

32. Ahmad KS, Qureshi R, Hameed M, Ahmad F, Nawaz T. Conservation assessment and medicinal importance of some plants resources from Sharda, Neelum Valley, Azad Jammu and Kashmir Pakistan. Int J Agricultural Biol 2012; 14(6):997-1000.

33. Tetik F, Civelek S, Cakilcioglu U. Traditional uses of some medicinal plants in Malatya (Turkey). J Ethnopharmacol. 2013;146(1):331-46.

34. Baydoun S, Chalak L, Dalleh H, Arnold N. Ethnopharmacological survey of medicinal plants used in traditional medicine by the communities of Mount Hermon, Lebanon. J Ethnopharmacol. 2015;173:139-56.

35. Ali S. Significance of flora with special reference to Pakistan. Pakistan J Botany. 2008:40(3):967-71.

36. ljaz F, Iqbal Z, Alam J, Khan SM, Afzal A, Rahman I, Afzal M, Islam M, Sohail M. Ethno medicinal study upon folk recipes against various human diseases in Sarban Hills, Abbottabad, Pakistan. World Journal of Zoology 2015; 10(1):41-46.

37. Ali H, Qaiser M. The ethnobotany of Chitral valley, Pakistan with particular reference to medicinal plants. Pakistan J Botany. 2009;41(4):2009-41.

38. Shinwari ZK, Qaiser M. Efforts on conservation and sustainable use of medicinal plants of Pakistan. Pakistan J Botany. 2011:43(1):5-10.

39. Bano A, Ahmad M, Saboor A, Hadda BT, Zafar M, Sultana S, Ashra MA. Quantitative ethnomedicinal study of plants used in the Skardu valley at high altitude of Karakoram-Himalayan range Pakistan. J Ethnobiol Ethnomed. 2014;10(10):43-71.

40. Qaseem MF, Qureshi R, Amjad MS, Waseem M, Sajid A. Ethnobotanical evaluation of tridational medicinal plants among thre rular communities of Goi and Dhanwa union council, District Kotli, Azad Jammu \& Kashmir. Appl Ecol Environm Res. 2019:6(2):340-9.

41. Amjad MS, Faisal Qaeem M, Ahmad I, Khan SU, Chaudhari SK, Malik NZ, Shaheen H, Khan AM. Descriptive study of plant resources in the context of the ethnomedicinal relevance of indigenous flora: A case study from Toli Peer National Park, Azad Jammu and Kashmir, Pakistan. PlosOne. 2017;12(2):e0171896.

42. Ullah M, Khan MU, Mahmood A, Malik RN, Hussain M, Wazir SM, Daud M, Shinwari ZK. An ethnobotanical survey of indigenous medicinal plants in Wana district south Waziristan agency, Pakistan. J Ethnopharmacol 2013, 150(3):918-924.

43. Khan MA, Khan MA, Hussain M, Ghulam GM. An ethnobotanical inventory of Himalayan region poonch valley azad kashmir (Pakistan). Ethnobotany Res Applications. 2010;8:107-23.

44. Ahmad KS, Hamid AF, Nawaz F, Hameed M, Ahmad F, Deng J, Mahroof S. Ethnopharmacological studies of indigenous plants in Kel village, Neelum Valley, Azad Kashmir. J Ethbiol Ethmed. 2017;13(168):1-8.

45. Anonymous. AJK at a Glance. - Pakistan Planning \& Development Department. Government of Azad Jammu and Kashmir, Muzaffarabad. 2007

46. Ahlaq R, Amjad MS, Qaseem MF, Fatima S, Chaudhari SK, Khan AM, Khan S, Malik NZ, Gardazi SMH, Bibi A. Saboon. Species diversity and vegetation structure from different climatic zones of tehsil Harighel, Bagh, Azad Kashmir, Pakistan analyzed through multivariate techniques. Appl Ecol Environmen Res. 2018;16(4):5193-211.

47. Shaheen H, Qaseem MF, Amjad MS, Bruschi P. Exploration of ethnomedicinal knowledge among rural communities of Pearl Valley; Rawalakot, District Poonch Azad Jammu and Kashmir. PlosOne. 2017;12(9):e0183956.

48. Edwards S, Nebel S, Heinrich M. Questionnaire surveys: methodological and epistemological problems for field-based ethnopharmacologists. Ethnopharmacol. 2005;100(1-2):30-6.

49. Kadam P, Bhalerao S. Sample size calculation. Int J Ayurveda Res. 2010:1(1):55.

50. Jain SK. Handbook of field and herbarium methods. New Delhi: Today \& Tomorrow; 1977.

51. Nasir E, Ali S, Stewart RR. Flora of West Pakistan: an annotated catalogue of the vascular plants of West Pakistan and Kashmir: Fakhri; 1972.

52. Nasir YJ, Ali S. Flora of Pakistan. Department of Botany, University of Karachi; National Herbarium; 1994-2010.

53. Chase MW, Christenhusz M, Fay M, Byng J, Judd WS, Soltis D, Mabberley D, Sennikov A, Soltis PS, Stevens PF. An update of the Angiosperm Phylogeny Group classification for the orders and families of flowering plants: APG IV Botanical J Linnean Soc. 2016;181:1-20.

54. Gardens RB, Kew MBG. The Plant List, Version 1.1. Recuperado el; 2013. p. 2.

55. Staub PO, Geck MS, Weckerle CS, Casu L, Leonti M. Classifying diseases and remedies in ethnomedicine and ethnopharmacology. Journal of Ethnopharmacology. 2015;174:514-9.

56. Heinrich M, Ankli A, Frei B, Weimann C, Sticher O. Medicinal plants in Mexico: Healers' consensus and cultural importance. Soci. Sci \& Med. 1998;4:1859-71.

57. Vijayakumar J, Yabesh M, Prabhu S, Manikandanz R, Muralidharan B. Quantitative ethnomedicinalstudy of plants used in the Nelliyampathy hills of Kerala, India. J. Ethpharmacol. 2015;161:238-254.

58. Ugulu I, Baslar S, Yorek N, Dogan Y. The investigation and quantitative ethnobotanical evaluation of medicinal plants used around Izmir province, Turkey. Journal of Medicinal plants research. 2009;3:345-67.

59. Alexiades MN, Sheldon JW. Selected guidelines for ethnobotanical research: a field manual. The New York Botanical Garden: Boranx, NY; 1996.

60. Gonza TM, Casares RPM, Sanchez RCP, Ramiro GJM, Molero MJ, Pieroni A. Medicinal plants in the Mediterranean area: synthesis of the results of the project RUBIA. Journal of Ethnopharmacoogyl. 2008;116(116):341-57.

61. Umair M, Altaf M, Abbasi AM. An ethnobotanical survey of indigenousmedicinal plants in Hafizabad district, Punjab- Pakistan. PlosOne 2017: 12(6):e0177912.

62. Giday M, Asfaw Z, Woldu Z. Medicinal plants of the Meinit ethnic group of Ethiopia: an ethnobotanical study. Journal of Ethnopharmacology. 2009; 124(124):513-21.

63. Tugume $P$, Esezah KK, Buyinza $M$, Namaalwa J, Kamatenesi $M$, Mucunguzi $P$, Kalema J. Ethnobotanical survey of medicinal plant species used by communities around Mabira Central Forest Reserve, Uganda. Journal of Ethnobiology and Ethnomedicine 2010; 12(5). 
64. Mahmood A, Malik RN, Shinwari ZK, Mahmood A. Ethnobotanical survey of plants from Neelum, Azad Jammu and Kashmir, Pakistan. Pakistan J Botany. 2011;43(105):10.

65. Khan S, Din NU, Sohail I, Rahman Fl, lqbal Z, Ali Z. Ethnobotanical study of some medicinal plants of Tehsil Kabal, District Swat, KP. Medicinal Aromatic Plants. 2015:4(189):2167-0412.10001.

66. Ch Ml, Ahmed F, Maqbool M, Hussain T. Ethnomedicinal inventory of flora of maradori valley, district forward Khahuta, Azad Kashmir, Pakistan. Am J Res Commun. 2013;1(6):239-61.

67. Amjad MS, Arshad M, Saboor A, Page S, Chaudhari SK. Ethnobotanical profiling of the medicinal flora of Kotli, Azad Jammu and Kashmir, Pakistan: Empirical reflections on multinomial logit specifications. Asian Pacific J Tropical Med. 2017:10(5):503-14.

68. Gilani SA, Qureshi RA, Gilani SJ. Indigenous uses of some important ethnomedicinal herbs of Ayubia National Park, Abbottabad, Pakistan. Ethnobotanical Leaflets. 2006;2006(1):32

69. Gulshan AB, Dasti AA, Hussain S, Atta Ml, Amin-ud-Din M. Indigenous uses of medicinal plants in rural areas of Dera Ghazi Khan, Punjab, Pakistan. ARPN J Agricultural Biological Sci. 2012;7(9):750-62.

70. Mahmood A, Mahmood A, Shaheen H, Qureshi RA, Sangi Y, Gilani SA. Ethno medicinal survey of plants from district Bhimber Azad Jammu and Kashmir, Pakistan. J Med Plants Res. 2011;5(11):2348-60.

71. Rana SK, Oli PS, Rana HK. Traditional botanical knowledge (TBK) on the use of medicinal plants in Sikles area, Nepal. Asian J Plant Sci Res. 2015;5(11):815.

72. Jadhav RR. Ethnobotanical and ethnomedicinal survey of Kadegaon Tahsil, Sangli (Maharashtra) India. J Medicinal Plants Stud. 2015;4(1):4.

73. Gidey $M$, Beyene $T$, Signorini MA, Bruschi P, Yirga G. Traditional medicinal plants used by Kunama ethnic group in Northern Ethiopia. J Medicinal Plants Res. 2015:9(15):494-509.

74. Dar EM. Ethnobotanical uses of plants of Lawat district Muzaffarabad, Azad Jammu \& Kashmir. Asian J Plant Sci. 2014;2(9):3.

75. Khan SW, Abbas Q, Hassan SN, Khan H, Hussain A. Medicinal plants of Turmic Valley (Central Karakoram National Park), Gilgit-Baltistan, Pakistan. J Bioresource Management. 2015;2(2):11.

76. Hussain W, Badshah L, Ullah M, Ali M, Ali A, Hussain F. Quantitative study of medicinal plants used by the communities residing in Koh-e-Safaid Range, northern Pakistani-Afghan borders. J Ethnobiol Ethnomed. 2018;14(1):30.

77. Ullah S, Bibi S. Ethnobotanical survey of medicinal plants of Musamina District Malakand Khyber Pukhtoonkhwa, Pakistan. Academic J Med Plants. 2018;6(6).

78. Umair M, Altaf M, Bussmann RW, Abbasi AM. Ethnomedicinal uses of the local flora in Chenab riverine area, Punjab province Pakistan. J Ethnobiol Ethnomed. 2019;15(1):7.

79. Akhtar N, Rashid A, Murad W, Bergmeier E. Diversity and use of ethnomedicinal plants in the region of Swat, North Pakistan. J Ethnobiol Ethnomed. 2013;9(1):25.

80. Kadir MF, Sayeed MSB, Setu NI, Mostafa A, Mia M. Ethnopharmacological survey of medicinal plants used by traditional health practitioners in Thanchi, Bandarban Hill Tracts, Bangladesh. J Ethnopharmacol. 2014;155(1): 495-508.

81. Jan G, Khan MA, Farhatullah JF, Ahmad M, Jan M, Zafar M. Ethnobotanical studies on some useful plants of Dir Kohistan valleys, KPK, Pakistan. Pakistan J Botany. 2011;43(4):1849-52.

82. Miller NJ, Ruiz-Larrea MB. Flavonoids and other plant phenols in the diet: their significance as antioxidants. J Nutritional Environmen Med. 2002;12(1):39-51.

83. Wickens GE, Field DV, Goodin JR. Plants for Arid Lands: Proceedings of the Kew International Conference on Economic Plants for Arid Lands Held in the Jodrell Laboratory, Royal Botanic Gardens, Kew, England, 23-27 July 1984: Springer Science \& Business Media; 2012.

84. Milliken W, Albert B, Gomez GG. Yanomami: a forest people: Royal Botanic Gardens, Kew; 1999.

85. Savoia D. Plant-derived antimicrobial compounds: alternatives to antibiotics. Future Microbiol. 2012;7(8):979-90.

86. Bradacs G, Heilmann J, Weckerle CS. Medicinal plant use in Vanuatu: a comparative ethnobotanical study of three islands. J Ethnopharmacol. 2011; 137(1):434-48

87. Leto C, Tuttolomondo T, La Bella S, Licata M. Ethnobotanical study in the Madonie Regional Park (Central Sicily, Italy)—Medicinal use of wild shrub and herbaceous plant species. J Ethnopharmacol. 2013;146(1):90-112.

88. Cornara L, La Rocca A, Marsili S, Mariotti M. Traditional uses of plants in the Eastern Riviera (Liguria, Italy). J Ethnopharmacol. 2009;125(1):16-30.
89. Neves JM, Matos C, Moutinho C, Queiroz G, Gomes LR Ethnopharmacological notes about ancient uses of medicinal plants in Trásos-Montes (northern of Portugal). J Ethnopharmacol. 2009;124(2):270-83.

90. Khan SM, Page S, Ahmad H, Shaheen H, Ullah Z, Ahmad M, Harper DM. Medicinal flora and ethnoecological knowledge in the Naran Valley, Western Himalaya, Pakistan. J Ethnobiol Ethnomed. 2013;9(1):4.

91. Zheng $X$, Xing F. Ethnobotanical study on medicinal plants around Mt. Yinggeling, Hainan Island, China. J Ethnopharmacol. 2009;124(2):197-210.

92. Panyaphu K, Van On T, Sirisa-Ard P, Srisa-Nga P, ChansaKaow S, Nathakarnkitkul S. Medicinal plants of the Mien (Yao) in Northern Thailand and their potential value in the primary healthcare of postpartum women. $J$ Ethnopharmacol. 2011;135(2):226-37.

93. Ghimire SK, Gimenez O, Pradel R, McKey D, Aumeeruddy-Thomas Y. Demographic variation and population viability in a threatened Himalayan medicinal and aromatic herb Nardostachys grandiflora: matrix modelling of harvesting effects in two contrasting habitats. J Appl Ecol. 2008;45(1):41-51.

94. Giday M, Asfaw Z, Elmqvist T, Woldu Z. An ethnobotanical study of medicinal plants used by the Zay people in Ethiopia. J Ethnopharmacol. 2003:85(1):43-52.

95. Ahmad M, Sultana S, Fazl-i-Hadi S, Ben Hadda T, Rashid S, Zafar M, Khan MA, Khan Ahmad M, Sultana S, Fazl-i-Hadi S, Ben Hadda T, Rashid S, Zafar M, Khan MA, Khan MPZ, Yaseen G. An Ethnobotanical study of Medicinal Plants in high mountainous region of Chail valley (District Swat-Pakistan). J Ethnobiol Ethnomed. 2014;10(1):36.

96. Inta A, Trisonthi P, Trisonthi C. Analysis of traditional knowledge in medicinal plants used by Yuan in Thailand. J Ethnopharmacol. 2013;149(1): 344-51.

97. El Amri J, El Badaoui K, Zair T, Bouharb H, Chakir S, Alaoui TEM. Ethnobotanical study of medicinal plants in the region El Hajeb (central Morocco). J Res Biol. 2015:4(8):1568-80.

98. Zhang $\mathrm{J}$, Cui M, He Y, Yu HL, Guo DA. Chemical fingerprint and metabolic fingerprint analysis of Danshen injection by HPLC-UV and HPLC-MS methods. J Pharmaceutical Biomedical Analysis. 2005;36(5):1029-35.

99. JA. An ethnobotanical study of medicinal plants used by tribal and native people of Madhupur forest area Bangladesh. J Ethnopharmacol 2014; 151(2):921-930.

100. Sanon S, Ollivier E, Azas N, Mahiou V, Gasquet M, Ouattara C, Nebie I, Traore A, Esposito F, Balansard G. Ethnobotanical survey and in vitro antiplasmodial activity of plants used in traditional medicine in Burkina Faso. J Ethnopharmacol. 2003;86(2-3):143-7.

101. Siew YY, Zareisedehizadeh S, Seetoh WG, Neo SY, Tan CH, Koh HL. Ethnobotanical survey of usage of fresh medicinal plants in Singapore. J Ethnopharmacol. 2014;155(3):1450-66.

102. Uddin MZ, Hassan MA. Determination of informant consensus factor of ethnomedicinal plants used in Kalenga forest, Bangladesh. Bangladesh J Plant Taxonomy. 2014;21(1):83-91.

103. Heinrich M, Edwards S, Moerman DE, Leonti M. Ethnopharmacological field studies: a critical assessment of their conceptual basis and methods. J Ethnopharmacol. 2009:124:1-17.

104. Ghorbani A, Langenberger G, Feng L, Sauerborn J. Ethnobotanical study of medicinal plants utilised by Hani ethnicity in Naban river watershed national nature reserve, Yunnan, China. J Ethnopharmacol. 2011;134(3):651-67.

105. Miraldi E, Ferri S, Mostaghimi V. Botanical drugs and preparations in the traditional medicine of West Azerbaijan (Iran). J Ethnopharmacol. 2001;75(2-3):77-87.

106. Mosaddegh M, Naghibi F, Moazzeni H, Pirani A, Esmaeili S. Ethnobotanical survey of herbal remedies traditionally used in Kohghiluyeh va Boyer Ahmad province of Iran. J Ethnopharmacol. 2012;141(1):80-95.

107. Tangjitman K, Wongsawad C, Kamwong K, Sukkho T, Trisonthi C. Ethnomedicinal plants used for digestive system disorders by the Karen of northern Thailand. J Ethnobiol Ethnomed. 2015:11(1):27.

108. Malla B, Gauchan DP, Chhetri RB. An ethnobotanical study of medicinal plants used by ethnic people in Parbat district of western Nepal. J Ethnopharmacol. 2015;165:103-17.

109. Murad W, Azizullah A, Adnan M, Tariq A, Khan KU, Waheed S, Ahmad A Ethnobotanical assessment of plant resources of Banda Daud Shah, district Karak, Pakistan. J Ehnobiol Ethnomed. 2013;9(1):77.

110. Adzu B, Amos S, Amizan M, Gamaniel K. Evaluation of the antidiarrhoeal effects of Zizyphus spina-christi stem bark in rats. ActaTtropica. 2003;87(2):245-50.

111. Schlage C, Mabula C, Mahunnah R. Heinrich. Medicinal plants of the Washambaa (Tanzania): documentation and ethnopharmacological evaluation. Plant Biol. 2000;2(1):83-92. 
112. Mukherjee PK, Nema NK, Venkatesh P, Debnath PK. Changing scenario for promotion and development of Ayurveda \pm way forward. J Ethnopharmacl. 2012;43(2):424-34.

113. Trotter R, Logan M, Trotter R, Logan M. Informant consensus: a new approach for identifying potentially effective medicinal plants. In: Etkin NL, editor. Plants and Indigenous Medicine and Diet - Behavioral Approaches 1986: Taylor and Francis.

114. Farooq A, Amjad MS, Ahmad K, Altaf M, Umair M, Abbasi AM. Ethnomedicinal knowledge of the rural communities of Dhirkot, Azad Jammu and Kashmir, Pakistan. J Ethnobiol Ethnomed. 2019;15(1):1-30.

115. Camou-Guerrero A, Reyes-García V, Martínez-Ramos M, Casas A. Knowledge and use value of plant species in a Rarámuri community: a gender perspective for conservation. Human Ecol. 2008:36(2):259-72.

116. Albuquerque UP, Lucena RF, Monteiro JM, Florentino AT, Cecília de Fátima C. Evaluating two quantitative ethnobotanical techniques. Ethnobotany Res Appl. 2006;:051-60.

117. Noreen F, Tamoor M, Adil M, Mushtaq U, Nisa Q. Data of ethnomedicinal plants in Wazirabad, District Gujranwala, Punjab. Pakistan J Pharma Care Health Syst. 2018;5(194):2376-0419.1000194.

118. Hassan-Abdallah A, Merito A, Hassan S, Aboubaker D, Djama M, Asfaw Z, Kelbessa E. Medicinal plants and their uses by the people in the Region of Randa, Dijbouti. J Ethnopharmacol. 2013;148(2):701-13.

119. Lulekal E, Kelbessa E, Bekele T, Yineger H. An ethnobotanical study of medicinal plants in Mana Angetu District, southeastern Ethiopia. J Ethnobiol Ethnomedicine. 2008;4(1):10.

120. Yineger $\mathrm{H}$, Yewhalaw D, Teketay D. Ethnomedicinal plant knowledge and practice of the Oromo ethnic group in southwestern Ethiopia. J Ethnobiol Ethnomed. 2008:4(1):11.

\section{Publisher's Note}

Springer Nature remains neutral with regard to jurisdictional claims in published maps and institutional affiliations.

Ready to submit your research? Choose BMC and benefit from:

- fast, convenient online submission

- thorough peer review by experienced researchers in your field

- rapid publication on acceptance

- support for research data, including large and complex data types

- gold Open Access which fosters wider collaboration and increased citations

- maximum visibility for your research: over $100 \mathrm{M}$ website views per year

At $\mathrm{BMC}$, research is always in progress.

Learn more biomedcentral.com/submissions 